\title{
Analytic-bilinear approach to integrable hierarchies. II. Multicomponent KP and 2D Toda lattice hierarchies.
}

\author{
L.V. Bogdanov* and B.G. Konopelchenko ${ }^{\dagger}$ \\ Consortium EINSTEIN \\ Dipartimento di Fisica dell'Università di Lecce \\ and Sezione INFN, 73100 Lecce, Italy
}

\begin{abstract}
Analytic-bilinear approach for construction and study of integrable hierarchies is discussed. Generalized multicomponent KP and 2D Toda lattice hierarchies are considered. This approach allows to represent generalized hierarchies of integrable equations in a condensed form of finite functional equations. Generalized hierarchy incorporates basic hierarchy, modified hierarchy, singularity manifold equation hierarchy and corresponding linear problems. Different levels of generalized hierarchy are connected via invariants of Combescure symmetry transformation. Resolution of functional equations also leads to the $\tau$-function and addition formulae to it.
\end{abstract}

${ }^{*}$ Permanent address: IINS, Landau Institute for Theoretical Physics, Kosygin str. 2, Moscow 117940, GSP1, Russia; e-mail Leonid@landau.ac.ru

${ }^{\dagger}$ Also: Budker Institute of Nuclear Physics, Novosibirsk 90, Russia

${ }^{\ddagger}$ European Institute for Nonlinear Studies via Transnationally Extended Interchanges 


\section{Introduction}

The Kadomtsev-Petviashvili (KP) equation and the whole KP hierarchy as well as the multicomponent KP and two-dimensional Toda lattice hierarchies are among the most important ingredients of the theory of integrable partial differential equations. They arise in various fields of physics from hydrodynamics to string theory.

Integrable hierarchies have been described and studied within the framework of different approaches. The Sato approach [1]- [6] and the $\bar{\partial}$-dressing method (see [7]- [9] and review [10]) are among the most beautiful and powerful among them. These two approaches look like completely different. The Sato approach is in essence algebraic with its infinite-dimensional Grassmannian, pseudo-differential operators, Hirota bilinear identity and $\tau$-function. In contrast, the $\bar{\partial}$-dressing method, based on the nonlocal $\bar{\partial}$-problem, mostly uses the technique of the complex variable theory.

Recently we proposed ([11], [12]) an approach which combines the characteristic features of both these methods, namely the Hirota bilinear identity from the Sato approach and analytic properties of the wavefunctions from the $\bar{\partial}$-dressing method. This analytic-bilinear approach is based on the generalized Hirota bilinear identity for the wave function $\psi(\lambda, \mu)$ with simple analytic properties (Cauchy-Baker-Akhiezer (CBA) function). The CBA function (or, to be more precise, its 'nonsingular part' $\chi(\lambda, \mu)$ is defined in some region $G$ of the complex plane and it is characterized by the generalized Hirota bilinear identity on the boundary of this region [13]. The CBA function arises both in $\bar{\partial}$-dressing method [8] and in finite gap integration method [14], and it can be introduced also to the context of general Grassmannian approach [6], [四].

According to generalized Hirota bilinear identity, the CBA function is a functional of the function $g$ defined on the boundary of $G$ (in fact, $g$ is a map of $\partial G$ to $\mathbf{C}^{\times}$(i.e. $\mathbf{C}-0$ ), or, in other words, it is an element of loop group). One can choose some special subgroups of the generic loop group parametrized by finite number of variables ('times') to get particular examples of integrable equations. This is a standard approach in the $\bar{\partial}$-dressing method. One may also try to use some generic loop group and extract a full infinite set of equations. So the notion of the hierarchy arises. The dependence of the CBA function on $g$ encodes the dynamics of the system. The hierarchy can be written down in variational form, i.e. as equations describing variations 
of the objects of theory under the arbitrary variation of group element $g$. To get an hierarchy in terms of times, one should introduce a parametrization of the loop group.

The distinctive feature of analytic-bilinear approach is that our primary object is the CBA function and hierarchies are written in the form of equations for the CBA function (we call these equations generalized hierarchies). The analytic-bilinear approach allows us to describe generalized hierarchies in a compact finite form. Such a generalized hierarchy contains basic integrable hierarchy, hierarchy of equation for wave functions (modified hierarchy) and hierarchy of equations for CBA-type wave functions (we call it singularity manifold equations (SM) hierarchy, because in the scalar KP case it coincides with the singularity manifold equations obtained from the Painleve analysis of KP; however, our contexts is completely different), and corresponding linear problems. The relation between three hierarchies of equations can be described in terms of invariants of Combescure symmetry group ([1], [12]).

The generalized Hirota bilinear identity provides various functional equations for the CBA function. The resolution of some of them leads to the introduction of the $\tau$-function, while others give rise to the addition theorems for the $\tau$-function. The properties of the $\tau$-function, such as the associated closed 1-form and its global definition are also arise in a simple manner within the analytic-bilinear approach.

The first part of this paper [12] was devoted to the scalar KP hierarchy. In this case $G$ is just a domain of the complex plane. Without the loss of generality, we choose $G$ as unit disc. The group element $g$ is defined on the unit circle and can be represented in a form

$$
g(\lambda)=\lambda^{n} g_{\text {in }} \times g_{\text {out }}
$$

where $n$ is the index of the function, $g_{\text {in }}$ and $g_{\text {out }}$ are functions analytic and having no zeroes respectively inside (outside) the unit circle on the complex plane (i.e. elements of the loop groups $\Gamma_{-}$and $\Gamma_{+}$). In the scalar KP hierarchy case, we have no opportunity to treat index variable without some additional construction, so we put index to zero. The functions $g_{\text {out }}$ and $g_{\text {in }}$ are parametrized in a standard way by infinite sets of 'positive' and 'negative' times. But it appears that in the scalar KP hierarchy case dependence of the objects of theory on $g_{\text {in }}$ (i.e. on negative times in our notations) is trivial, so the scalar KP hierarchy is defined effectively by the subgroup $\Gamma_{+}$of the loop group $\Gamma$. 
In the present second part of the paper we consider the generalized multicomponent KP hierarchy and the two-dimensional Toda lattice hierarchy (for other approaches to the usual multicomponent KP hierarchy and 2D Toda lattice hierarchy see [1], [2], [4], [5], [15], [16]).

There are two equivalent approaches to investigate multi-component hierarchy. One could start with the generalized Hirota identity for the matrixvalued CBA function. In this case $G$ is again a unit disc, and $g$ is a diagonal matrix function on the unit circle (i.e. a set of $N$ functions $g_{n}$ ). Another way is to start with the scalar CBA function defined in the region $G$ consisting of $N$ disconnected domains (unit discs for simplicity). In this case the dynamics is also defined by the set of $N$ functions $g_{n}$, and the CBA function consists, in fact, of $N \times N$ disconnected components. These two representations are exactly equivalent and are associated with the multicomponent loop group $\Gamma^{N}$. In our paper we will use both of them.

As in the scalar case, the objects of multicomponent KP theory depend trivially on the subgroup $\Gamma_{-}^{N}$. The difference with the scalar case consists in the presence of discrete variables (indices), though only the lattice vectors with zero general index are admissible.

Since the generalized Hirota bilinear identity is our primary object, it is quite natural to proceed from the hierarchy corresponding to the simplest choice of $G$ - the unit disk - to the hierarchies corresponding to $G$ consisting of several unit discs, i.e. from the scalar KP hierarchy to multicomponent $\mathrm{KP}$ hierarchy. But on the other hand, the loop group is also a fundamental object of the theory, so one would like to find an hierarchy with a full-scale action of generic loop group on the objects of theory. This hierarchy is known as Toda-lattice hierarchy. In our approach it can be introduced as a two-component KP hierarchy with the special choice of $G$ consisting of two discs with the common boundary (the unit circle): one disc around origin and one disc around infinity. In this case, up to some trivial dynamics, one can glue two special loops defining the two-component KP hierarchy to obtain a generic loop. In the case of Toda lattice hierarchy the discrete index variable, positive and negative time variables get their natural interpretation.

We first consider the multicomponent KP hierarchy. We start with the generalized Hirota identity for the matrix-valued CBA function and derive various finite functional equations for this function. Part of these functional equations present the compact form of the generalized multicomponent KP hierarchy which includes equations for potentials (the standard multicompo- 
nent KP hierarchy), equations for the wave functions (the modified multicomponent KP hierarchy), equations for CBA-type wave functions (singularity manifold equation hierarchy) and corresponding linear problems.

Resolution of functional equations introduces the set of $\tau$-functions. We discuss also the associated closed 1-form and its global definition. The simplest integrable systems are given by the resonantly interacting wave equations and by the scalar Darboux system which is its modified partner.

We present a compact form of equations of multicomponent KP hierarchy, modified KP hierarchy and singularity manifold equation hierarchy, using shift operators. Similar formulae for the first two cases can be found in [17].

The two-component case which corresponds to the generalized DaveyStewartson (DS) hierarchy is considered in detail.

The description of the generalized Toda lattice hierarchy within the analyticbilinear approach requires minor modifications of the multicomponent KP case. For simplicity we consider here only the Abelian Toda lattice hierarchy. It appears that modified Toda lattice equation (i.e. equation for the Toda lattice wave functions) coincides with the 2D Volterra system, which was recently discussed in literature in different contexts [18], [19]. We derive 2D Toda lattice singularity manifold equation, which was also discussed in [20]. The invariants of Combescure transformations group ([11], [12]) are found. We also present the hierarchies of equations using shift operators.

The paper is organized as follows. The generalized Hirota bilinear identity and some basic formulae are discussed in section 2. Generalized multicomponent KP hierarchy is considered in section 3. Compact forms of equations of generalized multicomponent KP hierarchy are presented in section 4. Section 5 is devoted to generalized DS hierarchy. The $\tau$-function for the DS hierarchy is introduced in section 6 . The $\tau$-function and corresponding addition formulae for the multicomponent KP hierarchy are considered in section 7 . Closed variational 1-form and global definition for the $\tau$-function are given in section 8. Basic equations for the generalized 2D Toda lattice hierarchy are derived in section 9. Concrete equations associated with the generalized 2DTL hierarchy are presented in section 10. Addition formulae for the 2DTL hierarchy are given in section 11. 


\section{Generalized Hirota identity, functional equa- tions and generalized multicomponent KP hierarchy}

The analytic-bilinear approach starts with the generalized Hirota bilinear identity

$$
\int_{\partial G} \chi\left(\nu, \mu ; g_{1}\right) g_{1}(\nu) g_{2}^{-1}(\nu) \chi\left(\lambda, \nu ; g_{2}\right) d \nu=0,
$$

where $\chi(\lambda, \mu ; g)$ is the CBA function normalized by the condition $\chi \rightarrow(\lambda-$ $\mu)^{-1}$ as $\lambda \rightarrow \mu$ and $\chi(\lambda, \mu)$ is analytic function of both variables $\lambda, \mu$ for $\lambda \neq \mu$ in the domain $G$ with the boundary $\partial G$. The quantity $g=g(\lambda, \mathbf{x})$ specifies the dependence on the infinite set of dynamical variables $\mathbf{x}=\left(x_{1}, x_{2}, \ldots\right)$. In terms of the wavefunction $\psi(\lambda, \mu ; g)$ defined via

$$
\psi(\lambda, \mu ; g)=g^{-1}(\mu) \chi(\lambda, \mu ; g) g(\lambda)
$$

the generalized Hirota bilinear identity looks like

$$
\int_{\partial G} \psi\left(\nu, \mu ; g_{1}\right) \psi\left(\lambda, \nu ; g_{2}\right) d \nu=0
$$

Note that the generalized Hirota bilinear identity is bilocal both in dynamical and spectral variables.

In this paper we consider the case for which $\chi$ and $\psi$ take their value in the full linear group GL(N,C), $G$ is a unit disc with a center $\lambda=0$. Elements $g(\lambda, \mathbf{x})$ belong to maximal abelian subgroup of $\mathrm{GL}(\mathrm{N}, \mathbf{C})$.

It is important to note that there is an alternative way to treat the matrix problem (1), starting from the scalar identity but with the region $G$ consisting of several disconnected domains. In this case one has to choose domain $G$ consisting of $\mathrm{N}$ unit discs $D_{\alpha}$ with the center at $\lambda=0$. Now the scalar CBA function effectively consists of $N \times N$ disconnected components and can be interpreted as matrix function defined on the unit disc:

$$
\chi_{\alpha \beta}(\lambda, \mu)=\chi(\lambda, \mu), \lambda \in D_{\beta}, \mu \in D_{\alpha} .
$$

The function $g$ in this framework is a set of $N$ functions defined on the unit circle. Thus, rewriting the scalar identity in matrix notations, we get exactly the same matrix generalized Hirota identity, where $g$ is a diagonal matrix. 
So the matrix structure in the scalar case is hidden in the multicomponent domain of definition of the CBA function. The advantage of the scalar treatment is the opportunity to preserve most of the structure of the onecomponent KP hierarchy case almost intact and to use geometric intuition in the matrix case. We will use both representations of the generalized Hirota identity in the paper.

The dependence on dynamical variables is hidden in the set of $N$ functions $g$ defined on the unit circle. The most natural choice would be to treat sets of arbitrary functions on the circle having no zeroes, forming the group by multiplication. Arbitrary $g$ can be represented in the form

$$
g(\lambda)=\lambda^{n} g_{\text {in }} \times g_{\text {out }}
$$

where discrete variable $n$ is the index of the function, $g_{\text {in }}$ and $g_{\text {out }}$ are functions analytic and having no zeroes respectively inside (outside) the unit circle on the complex plane. We will use the following parametrization for $g_{\text {in }}$ and $g_{\text {out }}$ :

$$
\begin{aligned}
& \left.g_{\alpha \text { out }}=\exp \left(\sum_{n=1}^{\infty} x_{(\alpha) n} \lambda^{-n}\right)\right) \\
& \left.g_{\alpha \text { in }}=\exp \left(\sum_{-\infty}^{n=-1} x_{(\alpha) n} \lambda^{-n}\right)\right) .
\end{aligned}
$$

In these formulae we have omitted 'the zero time' $x_{0}$, that defines simply some constant factor in front of $g$ and, according to (目), it doesn't change the function $\chi$.

So, in the general case the set of functions $g_{\alpha}$ can be parametrized by $N$ discrete times $n_{\alpha}$ and $N$ infinite sets of continuous variables $x_{\alpha n}, n \in \mathbf{Z}$. But in the case of KP hierarchy the dependence of the function $\chi(\lambda, \mu ; g)$ on $g_{\text {in }}$ (in other words, on the 'negative times') is trivial; according to (1) (the 'scalar' form), we get

$$
\chi\left(\lambda, \mu ; g \times g_{\text {in }}\right)=g_{\text {in }}^{-1}(\mu) \chi(\lambda, \mu ; g) g_{\text {in }}(\lambda) .
$$

It occurs that other important objects (KP solution, $\tau$-function) also depend on negative times trivially. Thus, for simplicity, from the very beginning we omit the dependence on the negative times.

As for discrete times, we will see, that the case of multicomponent KP hierarchy allows only sets of discrete times with the zero general index $\left(\sum_{\alpha=1}^{N} n_{\alpha}=0\right)$. 
So the case of multicomponent KP hierarchy seems a bit asymmetric with respect to positive and negative times, and it also doesn't allow to consider arbitrary lattice vectors of discrete times. The most natural way to resolve these problems is to consider multicomponent Toda-lattice hierarchy (see below). The objects of Toda-lattice hierarchy have a natural dependence on positive, negative and discrete times.

To find special solutions for (11), one may use some additional construction (e.g. $\bar{\partial}$-dressing method or algebro-geometric technique). But it is possible to develop a procedure of solving (1) in terms of (1) itself. In this case the function $\chi(\lambda, \mu, g=1)=\chi_{0}(\lambda, \mu)$ should be treated as initial data, and one can start from arbitrary $\chi_{0}(\lambda, \mu)$ possessing the necessary analytical properties. Then the dynamics of the function $\chi(\lambda, \mu)$ can be defined via the variational form of identity (1)

$$
\delta \chi(\lambda, \mu ; g)=\oint \chi(\nu, \mu ; g) \frac{\delta g(\nu)}{g(\nu)} \chi(\lambda, \nu ; g) d \nu .
$$

So the problem of solving (1) is reduced to (infinite number in terms of variables $x_{n}$ ) differential equations with initial condition. Taking the second variation in $\delta(\ln g)$, it is easy to prove that this system is self-consistent (i.e. the cross-derivatives are equal), and it is a correctly defined variational equation for the function $\chi(\lambda, \mu, g)$. Going further, one could take higher variational derivatives (all of them have a very simple form) and represent a formal solution in terms of Taylor-type series.

Another approach to solving (1) is to extract from it some linear integral equations. Indeed, let us choose some initial condition $\chi_{0}(\lambda, \mu)$. Then for the function $\chi(\lambda, \mu, g)$ from (1) we have a pair of equations

$$
\begin{gathered}
\int_{\partial G} \chi(\nu, \mu ; g) g(\nu) \chi_{0}(\lambda, \nu) d \nu=0, \\
\int_{\partial G} \chi_{0}(\nu, \mu) g^{-1}(\nu) \chi(\lambda, \nu ; g) d \nu=0 .
\end{gathered}
$$

These relations can be interpreted as linear integral equations for the function $\chi(\lambda, \mu ; g)$; integral equations of such type arise in the context of nonlocal Riemann problem. Using the technique developed in [13], one may show that if both of these equations are solvable (in the class of functions $\chi(\lambda, \mu)$ with the analytical properties specified above, here they play a role of normalization), then the solution for both equations is the same and it is unique. 
There is a very important class of functions $g$ corresponding to some special discrete transformations - meromorphyc functions - for which the dynamics can be found explicitly. Namely,the solution for both equations (9), (10) exists (and it is unique), if and only if meromorphic function $g$ has equal number of zeroes and poles in $G$ (taking into account the multiplicity), and it is given by the determinant formula [13]. This statement means that the dependence of $\chi$ on discrete (index) variables is correctly defined for the zero general index case and, moreover, it can be represented in explicit form [13].

\section{Generalized multicomponent KP hierarchy}

Proceeding with the multicomponent KP case, we choose the following parametrization for $g_{\text {out }}$ (matrix representation)

$$
g(\lambda, \mathbf{x})=\exp \left[\sum_{\alpha=1}^{N} \sum_{n=1}^{\infty} \frac{E_{\alpha}}{\lambda^{n}} x_{(\alpha) n}\right],
$$

where the matrices $E_{\alpha}$ form a basis of the commutative subalgebra of diagonal matrices: $\left(E_{\alpha}\right)_{\beta \gamma}=\delta_{\alpha \beta} \delta_{\beta \gamma} \quad(\alpha, \beta, \gamma=1, \ldots, N)$. So we have $N$ infinite series of dynamical variables $x_{(\alpha) n}$.

Equations (11) or (3) with $g$ of the form (11) generically define the multicomponent KP hierarchy. To extract from them finite functional equations similar to the scalar case we consider the Hirota identity (何) for the dynamical variables $x_{(\alpha) n}^{\prime}$ and $x_{(\alpha) n}$ related in a special way, namely

$$
x_{(\alpha) n}^{\prime}-x_{(\alpha) n}=\frac{1}{n} a_{\alpha}^{n}-\frac{1}{n} b_{\alpha}^{n},
$$

where $a_{\alpha}, b_{\alpha}(0 \geq \alpha \geq N)$ are arbitrary parameters. We will also use vector notations $\mathbf{x}=\left(\mathbf{x}_{\mathbf{1}}, \ldots, \mathbf{x}_{\mathbf{N}}\right), \mathbf{x}_{\alpha}=\left(x_{(\alpha) 1}, x_{(\alpha) 2}, \ldots\right)$. In this terms the preceding formula looks like

$$
\mathbf{x}_{(\alpha)}^{\prime}-\mathbf{x}_{(\alpha)}=\left[a_{\alpha}\right]-\left[b_{\alpha}\right]
$$

or

$$
\mathbf{x}^{\prime}-\mathbf{x}=[\mathbf{a}]-[b],
$$


where $[\mathbf{a}]_{(\alpha) i}=\frac{1}{i} a_{\alpha}^{i}$. For such $x$ and $x^{\prime}$ due to the formula $\log (1-\epsilon)=\sum_{n=1}^{\infty} \frac{\epsilon^{n}}{n}$ one has

$$
\left(g_{1}(\nu) g_{2}^{-1}(\nu)\right)_{\alpha \beta}=\delta_{\alpha \beta} \frac{\nu-a_{\alpha}}{\nu-b_{\alpha}} \quad 0 \geq \alpha, \beta \geq N .
$$

Substitution of the expression (15) into (1) gives

$$
\begin{aligned}
& \left(\frac{\mu-a_{\alpha}}{\mu-b_{\alpha}}\right) \chi_{\alpha \beta}(\lambda, \mu, \mathbf{x}+[\mathbf{a}])-\left(\frac{\lambda-a_{\beta}}{\lambda-b_{\beta}}\right) \chi_{\alpha \beta}(\lambda, \mu, \mathbf{x}+[\mathbf{b}])+ \\
& \sum_{\gamma=1}^{N}\left(b_{\gamma}-a_{\gamma}\right) \chi_{\gamma \beta}\left(\lambda, b_{\gamma}, \mathbf{x}+[\mathbf{a}]\right) \chi_{\alpha \gamma}\left(b_{\gamma}, \mu, \mathbf{x}+[\mathbf{b}]\right)=0 .
\end{aligned}
$$

Equation (16) represents the final functional form of the generalized multicomponent KP hierarchy. It encodes the usual multicomponent KP hierarchy and its modified version together with their linear problems. To demonstrate this fact we, similar to the scalar case [12], consider the particular form of equation (16) with $b_{\alpha}=0, \quad 0 \geq \alpha \geq N$

$$
\begin{aligned}
& \left(1-\frac{a_{\alpha}}{\mu}\right) \chi_{\alpha \beta}(\lambda, \mu, \mathbf{x}+[\mathbf{a}])-\left(1-\frac{a_{\beta}}{\lambda}\right) \chi_{\alpha \beta}(\lambda, \mu, \mathbf{x})= \\
& \sum_{\gamma=1}^{N} a_{\gamma} \chi_{\gamma \beta}(\lambda, 0, \mathbf{x}+[\mathbf{a}]) \chi_{\alpha \gamma}(0, \mu, \mathbf{x})=0,
\end{aligned}
$$

or, in terms of the CBA and Baker-Akhiezer functions

$$
\psi_{\alpha \beta}(\lambda, \mu, \mathbf{x}+[\mathbf{a}])-\psi_{\alpha \beta}(\lambda, \mu, \mathbf{x})=\sum_{\gamma=1}^{N} a_{\gamma} \psi_{\gamma \beta}(\lambda, \mathbf{x}+[\mathbf{a}]) \widetilde{\psi}_{\alpha \gamma}(\mu, \mathbf{x})(18)
$$

where $\psi_{\gamma \beta}(\lambda, \mathbf{x})=\chi(\lambda, 0, \mathbf{x}) g_{\beta}(\lambda), \widetilde{\psi}_{\gamma \beta}(\mu, \mathbf{x})=\chi(0, \mu, \mathbf{x}) g_{\gamma}^{-1}(\mu)$. The expansion of (18) in Taylor series in $a_{(\gamma)}$ gives rise in the first order to the following equation

$$
\frac{\partial \psi_{\alpha \beta}(\lambda, \mu, \mathbf{x})}{\partial x_{(\gamma) 1}}=\psi_{\gamma \beta}(\lambda, \mathbf{x}) \widetilde{\psi}_{\alpha \gamma}(\mu, \mathbf{x})
$$

Evaluating the equation (17) with $\alpha \neq \beta \neq \gamma \neq \alpha$ at $\lambda=\mu=0$, one obtains the system

$$
\frac{\partial P_{\alpha \beta}}{\partial \xi_{\gamma}}=P_{\alpha \gamma} P_{\gamma \beta}
$$


where $P_{\alpha \beta}=\chi_{\alpha \beta}(0,0, \mathbf{x}), \xi_{\gamma}=x_{(\gamma) 1}$, which is nothing but the Darboux system in terms of rotation coefficients [21] or resonantly interacting waves equation [7]. Equation (17) with $\alpha \neq \gamma$ and $\mu=0$ gives the system

$$
\frac{\partial \Psi_{\alpha}}{\partial \xi_{\gamma}}=P_{\alpha \gamma} \Psi_{\gamma},
$$

where

$$
\Psi_{\alpha}=\sum_{\beta=1}^{N} \oint d \lambda f_{\beta}(\lambda) \psi_{\alpha \beta}(\lambda, \mathbf{x})
$$

and $f_{\beta}(\lambda)$ are arbitrary functions. The system (21) is a well-known linear problem for equations $(20)$.

Equation (17) with $\beta \neq \gamma$ and $\lambda=0$ gives the adjoint linear problem for the system (20)

$$
\frac{\partial \widetilde{\Psi}_{\beta}}{\partial \xi_{\gamma}}=P_{\alpha \gamma} \widetilde{\Psi}_{\gamma},
$$

where $\widetilde{\Psi}_{\beta}=\sum_{\alpha=1}^{N} \oint d \mu \widetilde{f}_{\alpha}(\mu) \widetilde{\psi}_{\alpha \beta}(\mu, \mathbf{x})$ and $\widetilde{f}_{\alpha}(\mu)$ are arbitrary functions.

Further (21) implies that $P_{\alpha \gamma}=\frac{1}{\Psi_{\gamma}} \frac{\partial \Psi_{\alpha}}{\partial \xi_{\gamma}}$. Substituting this expression into (20), one gets

$$
\partial_{\beta} \partial_{\gamma} \Psi_{\alpha}=\Psi_{\beta}^{-1}\left(\partial_{\gamma} \Psi_{\beta}\right)\left(\partial_{\beta} \Psi_{\alpha}\right)+\Psi_{\gamma}^{-1}\left(\partial_{\beta} \Psi_{\gamma}\right)\left(\partial_{\gamma} \Psi_{\alpha}\right),
$$

where $\partial_{\beta}=\frac{\partial}{\partial \xi_{\beta}}$. This is a well-known Darboux-Zakharov-Manakov system which has been discovered in the theory of systems of conjugate surfaces first [21] and then has been rediscovered within the $\bar{\partial}$-dressing method in [7]. This system is a modified partner of the system (20). To get the linear problem for it, it is sufficient to substitute the expression $P_{\alpha \gamma}=\frac{1}{\Psi_{\gamma}} \frac{\partial \Psi_{\alpha}}{\partial \xi_{\gamma}}$ into (23).

Thus the system (17) contains the equation (20), its modified partner (24) and their linear problems.

The higher order terms in the expansion of (17) in $a_{\gamma}$ give rise to the higher analogues of equation (19). It is a straightforward check that these equations contain the higher order equations (20), their modified analogues and corresponding linear problems.

The generalized multicomponent KP hierarchy described by functional equation (16) has rather special structure. In particular, it contains $\mathrm{N}$ independent generalized scalar KP hierarchies. They are obviously given by 
equations (16) with $\alpha=\beta$ and $b_{\gamma}=a_{\gamma}$ for all $\gamma$ except $\gamma=\alpha$. It is also quite clear that the number of effective independent dynamical variables is equal to three. For instance, the systems (20) and (24) are overdetermined and represent a collection of commuting closed systems defined for every triad of dynamical variables.

Note also that one can get the multicomponent KP hierarchy starting with the scalar generalized Hirota identity (11). In this case one has to choose disconnected domain $G$ consisting of $\mathrm{N}$ unit discs $D_{\alpha}$ with the center at $\lambda=0$. In this case the scalar CBA function effectively consists of $N \times N$ disconnected components and can be interpreted as matrix function. So the matrix structure in the scalar case is hidden in the multicomponent domain of definition of the CBA function. The function $g(\lambda)$ is defined on the set of $N$ unit circles $S_{\alpha}$ and consists in fact of $N$ components $g_{\alpha}$ defined on a unit circle.

The advantage of the scalar treatment is the opportunity to preserve most of the structure of the one-component KP hierarchy case almost without modification.

Using the parametrization (5), we represent the function $g(\lambda)$ (defined on the set of $N$ unit circles $S_{\alpha}$ ) as

$$
g(\mathbf{x}, \lambda)=\prod_{\alpha=1}^{N} \exp \left(\sum_{i=1}^{\infty} x_{(\alpha) i} K_{\alpha}^{i}\right)
$$

where

$$
\begin{aligned}
K_{\alpha}(\lambda)=\frac{1}{\lambda}, & \lambda \in \mathbf{D}_{\alpha} \\
K_{\alpha}(\lambda)=0, & \lambda \notin \mathbf{D}_{\alpha} .
\end{aligned}
$$

In this case the transformation group element $g_{0}$ providing just the same shift of the $\mathbf{x}$ variables takes the form

$$
g_{0}(\nu)=\left(g_{1}(\nu) g_{2}^{-1}(\nu)\right)=\frac{\nu-a_{\alpha}}{\nu-b_{\alpha}} \quad \nu \in \mathbf{D}_{\alpha}
$$

The analogue of equation (16) reads

$$
\begin{aligned}
& g_{0}(\mu) \chi(\lambda, \mu, \mathbf{x}+[\mathbf{a}])-g_{0}(\lambda) \chi(\lambda, \mu, \mathbf{x}+[\mathbf{b}])+ \\
& \sum_{\gamma=1}^{N}\left(b_{\gamma}-a_{\gamma}\right) \chi\left(\lambda, b_{(\gamma)}, \mathbf{x}+[\mathbf{a}]\right) \chi\left(b_{\gamma}, \mu, \mathbf{x}+[\mathbf{b}]\right)=0 .
\end{aligned}
$$


It seems to be a scalar equation, but if we take into account that the function $\chi$ consists of a set of disconnected components and denote $\chi_{\alpha \beta}(\lambda, \mu)=$ $\chi(\lambda, \mu), \quad \lambda \in D_{\beta}, \mu \in D_{\alpha}$, then this equation gives rise exactly to the system (16).

The analogue of equation (17) is respectively

$$
\begin{aligned}
& \prod_{\alpha=1}^{N}\left(1-a_{\alpha} K_{\alpha}(\mu)\right) \chi(\lambda, \mu, \mathbf{x}+[\mathbf{a}])-\prod_{\alpha=1}^{N}\left(1-a_{\alpha} K_{\alpha}(\lambda)\right) \chi(\lambda, \mu, \mathbf{x})= \\
& \sum_{\gamma=1}^{N} a_{(\gamma)} \chi_{\gamma}(\lambda, \mathbf{x}+[\mathbf{a}]) \tilde{\chi}_{\gamma}(\mu, \mathbf{x})=0,
\end{aligned}
$$

and in terms of CBA and BA functions one gets

$$
\psi(\lambda, \mu, \mathbf{x}+[\mathbf{a}])-\psi(\lambda, \mu, \mathbf{x})=\sum_{\gamma=1}^{N} a_{\gamma} \psi_{\gamma}(\lambda, \mathbf{x}+[\mathbf{a}]) \widetilde{\psi}_{\gamma}(\mu, \mathbf{x}),
$$

where we have introduced notations

$$
\begin{aligned}
& \chi_{\gamma}(\lambda, \mathbf{x})=\chi\left(\lambda, 0_{\gamma}, \mathbf{x}\right), \\
& \tilde{\chi}_{\gamma}(\mu, \mathbf{x})=\chi\left(0_{\gamma}, \mu, \mathbf{x}\right), \\
& \psi_{\alpha}(\lambda, \mathbf{x})=g(\lambda) \chi_{\alpha}(\lambda, \mathbf{x}), \\
& \widetilde{\psi}_{\alpha}(\mu, \mathbf{x})=g^{-1}(\mu) \widetilde{\chi}_{\alpha}(\mu, \mathbf{x}),
\end{aligned}
$$

$0_{\alpha}$ denotes the center point of the disc $D_{\alpha}$.

We will also use following notations, most of them exactly corresponding to notations introduced in frame of matrix representation:

for potentials

$$
\begin{gathered}
\chi_{\alpha \beta}(\mathbf{x})=\chi_{\mathrm{r}}\left(0_{\beta}, 0_{\alpha}, \mathbf{x}\right), \\
\chi_{\mathrm{r}}(\lambda, \mu, \mathbf{x})=\chi(\lambda, \mu, \mathbf{x})-\delta_{\alpha \beta} \frac{1}{\lambda-\mu}, \quad \lambda \in D_{\alpha}, \mu \in D_{\beta}
\end{gathered}
$$

for wave functions

$$
\begin{aligned}
& \Phi(\mathbf{x})=\iint_{\partial G} d \lambda d \mu f(\lambda) \widetilde{f}(\mu) \psi(\lambda, \mu, \mathbf{x}) \\
& \Psi_{\alpha}(\mathbf{x})=\int_{\partial G} d \lambda f(\lambda) \psi_{\alpha}(\lambda, \mathbf{x}) \\
& \widetilde{\Psi}_{\alpha}(\mathbf{x})=\int_{\partial G} d \mu \widetilde{f}(\mu) \widetilde{\psi}_{\alpha}(\mu, \mathbf{x})
\end{aligned}
$$

where $f(\lambda), \tilde{f}(\mu)$ are arbitrary functions defined on the boundary of $G$ (i.e. on $N$ unit circles). 


\section{Equations of generalized KP hierarchy in compact form}

The basic equation of the multicomponent KP hierarchy (17) looks very simple and contains all the information about generalized hierarchy - hierarchy of linear problems and hierarchy of equations. However, sometimes this information could be excessive and one would prefer to have a compact form of the equations of the hierarchy. As we have demonstrated in the previous section in the case of DS hierarchy, it is possible to extract all the hierarchy of equations from (17) in terms of shift operators. To derive the equations of the KP hierarchy, we will use generic shifts of times. For each triad of different shifts (i.e. shifts with different parameters) it is possible to derive a closed system of equations, representing, in fact, the multicomponent KP hierarchy.

As we will see later, the multicomponent KP hierarchy in a general case incorporates also discrete times not reducible to the shifts of continuous times. So equations of multicomponent KP hierarchy can also contain these essentially discrete times. It is possible to include them to equations of this section, but now we will derive the equations of KP hierarchy containing only continuous times. Discrete times will be discussed later in the context of Toda lattice hierarchy.

Thus, we will derive a compact form of the equation of multicomponent KP hierarchy, modified KP hierarchy and singularity manifold equation hierarchy. It is interesting to note that these equations are obtained in fact in the form of lattice equations, and all the hierarchy is given by the expansion of these lattice equations with respect to the parameters of the lattice.

In this section we will use the matrix form of the equation (17), i.e.

$$
\Delta \chi(\lambda, \mu)+\frac{1}{\lambda} \chi(\lambda, \mu) A-\frac{1}{\mu} A \cdot T \chi(\lambda, \mu)=\chi(0, \mu) A T \chi(\lambda, 0) .
$$

Here the common argument $\mathbf{x}$ is omitted, the function $\chi(\lambda, \mu, \mathbf{x})$ is $N \times N$

matrix-valued function, $A$ is a diagonal matrix, $A_{i j}=\delta_{i j} a_{i}$, and we have introduced shift operator and difference operator

$$
\begin{aligned}
& T \chi(\lambda, \mu, \mathbf{x})=\chi(\lambda, \mu, \mathbf{x}+[\mathbf{a}]), \\
& \Delta \chi(\lambda, \mu, \mathbf{x})=(T-1) \chi(\lambda, \mu, \mathbf{x}) .
\end{aligned}
$$


If one takes the first term of expansion of the relation (32) at $\lambda=0, \mu=0$, one obtains an identity containing the potential $u=\chi_{\mathrm{r}}(0,0, \mathbf{x})$ and two higher terms of expansion of $\chi_{\mathrm{r}}(\lambda, \mu, \mathbf{x})$. It is possible to cancel these terms by combining three equations (32) with different shift parameters $\mathbf{a}_{\mathbf{k}}, k \in$ $\{1,2,3\}$ and get an equation for the potential $u$.

First, it is easy to cancel the terms containing $A_{i} / \lambda$ combining pairs of equations

$$
\begin{aligned}
& \Delta_{i} \chi(\lambda, \mu) A_{j}-\Delta_{j} \chi(\lambda, \mu) A_{i}+\frac{1}{\mu}\left(A_{i} T_{i} \chi(\lambda, \mu) A_{j}-A_{j} T_{j} \chi(\lambda, \mu) A_{i}\right)= \\
& \chi(0, \mu) A_{i} T_{i} \chi(\lambda, 0) A_{j}-\chi(0, \mu) A_{j} T_{j} \chi(\lambda, 0) A_{i} .
\end{aligned}
$$

And then, multiplying this equation by the operator $A_{k} T_{k}$ from the left, taking sum by different permutations of indices $i, j, k$ and using the first term of expansion by $\lambda, \mu$ at $\lambda=0, \mu=0$, one obtains the equation

$$
\sum_{(i j k)} \epsilon_{i j k} A_{k} T_{k}\left(\Delta_{i} u-u A_{i} T_{i} u\right) A_{j}=0
$$

where $u=\chi_{\mathrm{r}}(0,0, \mathbf{x})$, summation goes over different permutations of indices. This extremely simple equation generates all equations of multicomponent KP hierarchy (not containing essentially discrete variables). Similar equation can be found in [17].

The scalar case of (34) represents a scalar KP hierarchy in the form of superposition formula for three Bäcklund transformations. To simplify the derivation of equations of KP hierarchy from this equation, it is possible to take the first order of expansion in terms of the parameter $a_{1}$, performing, in fact, a transition $\Delta_{1} \rightarrow \frac{\partial}{\partial x_{1}}, T_{1} \rightarrow 1$ and thus distinguishing the continuous variable $x:=x_{1}$ (obtaining, in fact, superposition formula for two Bäcklund transformations).

It is easy to check that the choice of three shifts $T_{i}: \mathbf{x}_{\mathbf{i}} \rightarrow \mathbf{x}_{\mathbf{i}}+\left[a_{i}\right]$ in the formula (34) in the first nontrivial order of expansion $\left(a_{i} a_{j} a_{k}\right)$ leads to the standard form of three waves equation.

Using cross-differences of equations (32), it is possible to obtain equations for the wave functions ('modified equations')

$$
\sum_{(i j k)} \epsilon_{i j k} T_{k}\left(\left(A_{j} T_{i} \widetilde{\Psi}\right) \cdot \widetilde{\Psi}^{-1}\right) A_{k}=0 .
$$


Equations of this type for the DS case and N-wave equations case were derived in [22], see also the lattice form in [17].

And finally, it is easy to check using (34) that $\Phi$ satisfies the equation

$$
\left(T_{2} \Delta_{1} \Phi\right) \cdot\left(T_{2} \Delta_{3} \Phi\right)^{-1} \cdot\left(T_{3} \Delta_{2} \Phi\right) \cdot\left(T_{3} \Delta_{1} \Phi\right)^{-1} \cdot\left(T_{1} \Delta_{3} \Phi\right) \cdot\left(T_{1} \Delta_{2} \Phi\right)^{-1}=1 .
$$

This symmetric equation represents a multicomponent singularity manifold equation hierarchy. In the scalar case it reads

$$
\left(T_{2} \Delta_{1} \Phi\right)\left(T_{3} \Delta_{2} \Phi\right)\left(T_{1} \Delta_{3} \Phi\right)=\left(T_{2} \Delta_{3} \Phi\right)\left(T_{3} \Delta_{1} \Phi\right)\left(T_{1} \Delta_{2} \Phi\right) .
$$

We would like to remind here the main points of the concept of Combescure symmetry transformation. The term Combescure symmetry transformation originates from geometry, where it is used for the transformation of surface, keeping all tangent vectors parallel [21]. For the integrable Darboux system describing triply conjugate systems of surfaces this transformation has a very simple interpretation in the contexts of theory of integrable systems, and in fact a general class of integrable equations ('modified' equations) possesses this type of transformation (though the geometrical interpretation in this case may be missing). Our concept of the Combescure transformation is inspired mainly by integrability, not geometry (see [11]).

Integrable equations for the wave functions (i.e. modified equations and singularity manifold type equations) possess the symmetry transformations corresponding to the freedom of choice of the wave function for the same CBA function. In spectral terms this symmetry is described by the change of weight functions $\rho(\lambda), \eta(\mu)$. In terms of equations themselves this symmetry is represented using the Combescure invariants. The solutions are connected by the Combescure symmetry transformation, if Combescure invariants for them are the same. The Combescure symmetry group consists of two subgroups ('left' and 'right'), corresponding to the change of one weight function $(f(\lambda)$ or $\widetilde{f}(\mu)$, see notations (31); in our case $f(\lambda)$ and $\widetilde{f}(\mu)$ are matrix-valued functions, multiplication by $f(\lambda)$ is from the right, by $\widetilde{f}(\mu)$ is from the left, and here we will use the terms 'left' and 'right' Combescure invariants according to the rules of matrix multiplication). Singularity manifold type equations possess three types of invariants: left, right and complete invariants. Modified equations possess only one type of invariants (left or right) and are invariant under opposite Combescure subgroup. In this sense the basic hierarchy is invariant under the action of all Combescure group. 
We will write down some of the Combescure invariants of equations (35), (36). Combescure invariants for the equation (35) read

$$
C_{i j}=\left(\Delta_{i} \widetilde{\Psi} A_{j}-\Delta_{j} \widetilde{\Psi} A_{i}\right)(\widetilde{\Psi})^{-1} .
$$

Left Combescure invariants for the equation (36) are given by

$$
C_{i j}^{\prime}=\left(\Delta_{j} \Phi\right)^{-1}\left(\Delta_{i} \Phi\right) .
$$

\section{Generalized DS hierarchy}

Now we will consider the 2-component case of multicomponent KP hierarchy in more detail. In this section we derive the DS hierarchy and the hierarchy of corresponding linear problems, the mDS hierarchy and linear problems, and in the end we present the compact form of the hierarchy of Combescure transformation invariants for the $\mathrm{mDS}$ hierarchy. To perform all the derivations in terms of hierarchy, we use the formulae containing two continuous times $\left(x_{1}:=x_{(1) 1}\right.$ and $x_{2}=: x_{(2) 1}$ and discrete shifts $\left[a_{1}\right]$ and $\left[a_{2}\right]$, the first acting on $\mathbf{x}_{(\mathbf{1})}$, the second on $\mathbf{x}_{(\mathbf{2})}$. These shifts change all the times of the hierarchy, and the expansion in $a_{1}, a_{2}$ will generate all the hierarchy in terms of partial differential equations.

To derive DS hierarchy and linear problems, we will use the following special cases of the formula (28)

$$
\begin{aligned}
& \frac{1}{a_{1}} \Delta_{1} \chi(\lambda, \mu, \mathbf{x})+K_{1}(\lambda) \chi(\lambda, \mu, \mathbf{x})-K_{1}(\mu) T_{1} \chi(\lambda, \mu, \mathbf{x}) \\
& =\widetilde{\chi}_{1}(\mu, \mathbf{x}) T_{1} \chi_{1}(\lambda, \mathbf{x}), \\
& \frac{1}{a_{2}} \Delta_{2} \chi(\lambda, \mu, \mathbf{x})+K_{2}(\lambda) \chi(\lambda, \mu, \mathbf{x})-K_{2}(\mu) T_{2} \chi(\lambda, \mu, \mathbf{x}) \\
& =\widetilde{\chi}_{2}(\mu, \mathbf{x}) T_{2} \chi_{2}(\lambda, \mathbf{x}), \\
& \partial_{1} \chi(\lambda, \mu, \mathbf{x})+\left(K_{1}(\lambda)-K_{1}(\mu)\right) \chi(\lambda, \mu, \mathbf{x})=\widetilde{\chi}_{1}(\mu, \mathbf{x}) \chi_{1}(\lambda, \mathbf{x}), \\
& \partial_{2} \chi(\lambda, \mu, \mathbf{x})+\left(K_{2}(\lambda)-K_{2}(\mu)\right) \chi(\lambda, \mu, \mathbf{x})=\widetilde{\chi}_{2}(\mu, \mathbf{x}) \chi_{2}(\lambda, \mathbf{x}),
\end{aligned}
$$

where we have introduced shift operators and difference operators

$$
\begin{aligned}
& T_{1} \chi\left(\lambda, \mu, \mathbf{x}_{\mathbf{1}}, \mathbf{x}_{\mathbf{2}}\right)=\chi\left(\lambda, \mu, \mathbf{x}_{\mathbf{1}}+\left[a_{1}\right], \mathbf{x}_{\mathbf{2}}\right), \\
& T_{2} \chi\left(\lambda, \mu, \mathbf{x}_{\mathbf{1}}, \mathbf{x}_{\mathbf{2}}\right)=\chi\left(\lambda, \mu, \mathbf{x}_{\mathbf{1}}, \mathbf{x}_{\mathbf{2}}+\left[a_{2}\right]\right), \\
& \Delta_{1} \chi\left(\lambda, \mu, \mathbf{x}_{\mathbf{1}}, \mathbf{x}_{\mathbf{2}}\right)=\left(T_{1}-1\right) \chi\left(\lambda, \mu, \mathbf{x}_{\mathbf{1}}, \mathbf{x}_{\mathbf{2}}\right), \\
& \Delta_{2} \chi\left(\lambda, \mu, \mathbf{x}_{\mathbf{1}}, \mathbf{x}_{\mathbf{2}}\right)=\left(T_{2}-1\right) \chi\left(\lambda, \mu, \mathbf{x}_{\mathbf{1}}, \mathbf{x}_{\mathbf{2}}\right) .
\end{aligned}
$$


These operators can also be represented as differential operators

$$
\begin{aligned}
& T_{1}=\exp \left(\sum_{n=1}^{\infty} \frac{a_{1}^{n}}{n} \partial_{(1) n}\right), \\
& T_{2}=\exp \left(\sum_{n=1}^{\infty} \frac{a_{2}^{n}}{n} \partial_{(2) n}\right) .
\end{aligned}
$$

The coefficient of expansion of $T_{1}$ in $a_{1}, T_{2}$ in $a_{2}$ are given explicitly in terms of Schur's polynomials $p_{N}\left(\frac{\partial_{(1) 1}}{1}, \ldots, \frac{\partial_{(1) N}}{N}\right), p_{N}\left(\frac{\partial_{(2) 1}}{1}, \ldots, \frac{\partial_{(2) N}}{N}\right)$

The L-operator for the DS hierarchy is obtained by taking (42) at $\lambda=0_{2}$, (43) at $\lambda=0_{1}$ (and then passing on to the wave functions (311)

$$
\begin{array}{ll}
\partial_{1} \Psi_{2}=u \Psi_{1}, & u=\chi_{21}(\mathbf{x}) ; \\
\partial_{2} \Psi_{1}=v \Psi_{2}, & v=\chi_{12}(\mathbf{x}) ;
\end{array}
$$

while the hierarchy of A operators is obtained by taking respectively (40) at $\mu=0_{2}$, (41) at $\mu=0_{1}$

$$
\begin{aligned}
& \frac{\Delta_{1}}{a_{1}} \Psi_{2}=u T_{1} \Psi_{1}, \\
& \frac{\Delta_{2}}{a_{2}} \Psi_{1}=v T_{2} \Psi_{2} .
\end{aligned}
$$

One can also obtain dual $\mathrm{L}$ and $\mathrm{A}$ operators, taking (40), (42) at $\mu=0_{2}$, (411), (43) at $\lambda=0_{1}$.

It is possible to get DS hierarchy through compatibility conditions, but we prefer to extract it directly from the basic formulae (40), (41), (42), (43).

Taking the difference of equations (40), (42) at $\lambda=0_{1}, \mu=0_{2}$, and also using similar combinations, one obtains the system of equations

$$
\begin{aligned}
& \left(1 / a_{1}\left(T_{1}-1\right)-\partial_{1}\right) u=\left(T_{1}-1\right) \chi_{11} u, \\
& \left(1 / a_{1}\left(1-T_{1}^{-1}\right)-\partial_{1}\right) v=\left(T_{1}^{-1}-1\right) \chi_{11} v, \\
& \left(1 / a_{2}\left(T_{2}-1\right)-\partial_{2}\right) v=\left(T_{2}-1\right) \chi_{22} v, \\
& \left(1 / a_{2}\left(1-T_{2}^{-1}\right)-\partial_{2}\right) u=\left(T_{2}^{-1}-1\right) \chi_{22} u, \\
& \partial_{1} \chi_{22}=u v \\
& \partial_{2} \chi_{11}=u v .
\end{aligned}
$$


This system of equations represent a compact form of DS hierarchy. The expansion of this systems in $a_{1}, a_{2}$ generates the DS equation and higher equations of the hierarchy. It is useful to note that this expansion has the structure of recursion relations, expressing the derivatives in higher times through derivatives in lower times.

It is possible to obtain DS hierarchy from the formula (34). One could start with the three shifts $T_{1}: \mathbf{x}_{\mathbf{1}} \rightarrow \mathbf{x}_{\mathbf{1}}+\left[a_{1}\right], T_{2}: \mathbf{x}_{\mathbf{2}} \rightarrow \mathbf{x}_{\mathbf{2}}+\left[a_{2}\right], T_{3}:$ $\mathbf{x}_{\mathbf{1}} \rightarrow \mathbf{x}_{\mathbf{1}}+\left[a_{3}\right]$ (or $T_{3}: \mathbf{x}_{\mathbf{2}} \rightarrow \mathbf{x}_{\mathbf{2}}+\left[a_{3}\right]$ for the part of hierarchy with higher times connected with the disc $D_{2}$ ). Then, taking the first order of expansion in $a_{1}, a_{2}$ and thus performing a transition from two difference operators to derivatives $\Delta_{1} \rightarrow \frac{\partial}{\partial x_{1}}, T_{1} \rightarrow 1, \Delta_{2} \rightarrow \frac{\partial}{\partial x_{2}}, T_{2} \rightarrow 1$ and finally writing down matrix components of equation (34), one obtains DS hierarchy exactly in the form (46).

The zero order of expansion just gives trivial identities. The first order of expansion of the first pair of equations in $a_{1}$, the second pair in $a_{2}$ gives the DS system

$$
\begin{aligned}
& \frac{1}{2}\left(\partial_{(1) 2}+\partial_{1}^{2}\right) u=u \partial_{1} \chi_{11}, \\
& \frac{1}{2}\left(\partial_{(1) 2}-\partial_{1}^{2}\right) v=-v \partial_{1} \chi_{11}, \\
& \frac{1}{2}\left(\partial_{(2) 2}+\partial_{2}^{2}\right) v=v \partial_{2} \chi_{22}, \\
& \frac{1}{2}\left(\partial_{(2) 2}-\partial_{2}^{2}\right) u=-u \partial_{2} \chi_{22}, \\
& \partial_{1} \chi_{22}=u v \\
& \partial_{2} \chi_{11}=u v
\end{aligned}
$$

where $\partial_{(1) 2}=\frac{\partial}{\partial x_{(1) 2}}, \partial_{(2) 2}=\frac{\partial}{\partial x_{(2) 2}}$. The usual form of the DS system (see e.g. [23]) is a closed subsystem of (47) written in terms of the time $t$ defined as $\partial_{t}=\partial_{(1) 2}+\partial_{(2) 2}$.

The second order of expansion, with the use of the DS equations (47), gives

$$
\begin{aligned}
& \frac{1}{3}\left(\partial_{(1) 3}-\partial_{1}^{3}\right) u=u \partial_{2}^{-1} \partial_{1}\left(u \partial_{1} v\right)-\partial_{1}\left(u \partial_{2}^{-1} \partial_{1} u v\right), \\
& \frac{1}{3}\left(\partial_{(1) 3}-\partial_{1}^{3}\right) v=v \partial_{2}^{-1} \partial_{1}\left(v \partial_{1} u\right)-\partial_{1}\left(v \partial_{2}^{-1} \partial_{1} u v\right), \\
& \frac{1}{3}\left(\partial_{(2) 3}-\partial_{2}^{3}\right) v=v \partial_{1}^{-1} \partial_{2}\left(v \partial_{2} u\right)-\partial_{2}\left(v \partial_{1}^{-1} \partial_{2} u v\right), \\
& \frac{1}{3}\left(\partial_{(2) 3}-\partial_{2}^{3}\right) u=u \partial_{1}^{-1} \partial_{2}\left(u \partial_{2} v\right)-\partial_{2}\left(u \partial_{1}^{-1} \partial_{2} u v\right),
\end{aligned}
$$


which is the higher DS system $([24)$. The reduction $u=1$ gives rise to the split Nizhnik-Veselov-Novikov equation for $v$ [25], [26], while the reduction $u=v$ leads to the modified Nizhnik-Veselov-Novikov equation [24].

To proceed to modified DS hierarchy case, we integrate equations (40), (41), (42), (43) over $\lambda, \mu$ with some weight functions and obtain equations for wave functions

$$
\begin{aligned}
& \frac{\Delta_{1}}{a_{1}} \Phi=\widetilde{\Psi}_{1} T_{1} \Psi_{1}, \\
& \frac{\Delta_{2}}{a_{2}} \Phi=\widetilde{\Psi}_{2} T_{2} \Psi_{2}, \\
& \partial_{1} \Phi=\widetilde{\Psi}_{1} \Psi_{1}, \\
& \partial_{2} \Phi=\widetilde{\Psi}_{2} \Psi_{2} .
\end{aligned}
$$

The L operator for modified DS hierarchy can be obtained from the last pair of equations, using equations (44) and dual DS L-operator

$$
\partial_{1} \partial_{2} \Phi=\left(\partial_{2} U\right) \partial_{1} \Phi+\left(\partial_{1} V\right) \partial_{2} \Phi
$$

where $U=\ln \Psi_{1}, V=\ln \Psi_{2}$. The hierarchy of A operators arises if one uses the second pair of equations (50) to exclude $\widetilde{\Psi}_{1}, \widetilde{\Psi}_{2}$ from the first pair

$$
\begin{array}{ll}
\frac{\Delta_{1}}{a_{1}} \Phi=W_{1} \partial_{1} \Phi, & W_{1}=\frac{T_{1} \Psi_{1}}{\Psi_{1}} ; \\
\frac{\Delta_{2}}{a_{2}} \Phi=W_{2} \partial_{2} \Phi, & W_{2}=\frac{T_{2} \Psi_{2}}{\Psi_{2}} .
\end{array}
$$

The compatibility condition for (51), (52) gives the modified DS hierarchy

$$
\begin{aligned}
& \frac{1}{a_{1}} \Delta_{1} \partial_{2} U+\left(T_{1} \partial_{2} U\right)\left(\partial_{1} W_{1}\right)+\left(T_{1} \partial_{1} V\right)\left(\partial_{2} W_{1}\right)+W_{1}\left(\partial_{2} U\right) \Delta_{1} \partial_{1} V= \\
& \partial_{1} \partial_{2} W_{1}+\partial_{1}\left(W_{1} \partial_{2} U\right), \\
& \frac{1}{a_{1}} \Delta_{1} \partial_{1} V+W_{1}\left(\Delta_{1} \partial_{1} V\right)\left(\partial_{1} V\right)-\partial_{1}\left(W_{1} \partial_{1} V\right)=0, \\
& W_{1}=\exp \left(\Delta_{1} U\right),
\end{aligned}
$$

and for times with the subscript (2)

$$
\frac{1}{a_{2}} \Delta_{2} \partial_{1} V+\left(T_{2} \partial_{1} V\right)\left(\partial_{2} W_{2}\right)+\left(T_{2} \partial_{2} U\right)\left(\partial_{1} W_{2}\right)+W_{2}\left(\partial_{1} V\right) \Delta_{2} \partial_{2} U=
$$




$$
\begin{aligned}
& \partial_{2} \partial_{1} W_{2}+\partial_{2}\left(W_{2} \partial_{1} V\right), \\
& \frac{1}{a_{2}} \Delta_{2} \partial_{2} U+W_{2}\left(\Delta_{2} \partial_{2} U\right)\left(\partial_{2} U\right)-\partial_{2}\left(W_{2} \partial_{2} U\right)=0, \\
& W_{2}=\exp \left(\Delta_{2} V\right) .
\end{aligned}
$$

The zero order of expansion of these equations in $a_{1}, a_{2}$ gives modified DS equation 22

$$
\begin{aligned}
& \frac{1}{2} \partial_{2}\left(\partial_{(1) 2} U-\partial_{1}^{2} U-\left(\partial_{1} U\right)^{2}\right)+\partial_{1}\left(\partial_{2} U \cdot \partial_{1} V\right)=0 \\
& \partial_{(1) 2} V+\partial_{1}^{2} V+\left(\partial_{1} V\right)=\partial_{1} V \cdot \partial_{1} U \\
& \frac{1}{2} \partial_{1}\left(\partial_{(2) 2} V-\partial_{2}^{2} V-\left(\partial_{2} V\right)^{2}\right)+\partial_{2}\left(\partial_{2} U \cdot \partial_{1} V\right)=0 \\
& \partial_{(2) 2} U+\partial_{2}^{2} U+\left(\partial_{2} U\right)^{2}=\partial_{2} V \cdot \partial_{2} U
\end{aligned}
$$

The hierarchy of Combescure invariants ([11]) for the modified DS hierarchy is obtained by representation of the $\mathrm{DS}$ solutions $u, v$ through the $\mathrm{mDS}$ solutions $U, V$

$$
\begin{aligned}
& u=\exp \left(-T_{1} U\right) \frac{\Delta_{1}}{a_{1}} \exp (V), \\
& v=\exp \left(-T_{2} V\right) \frac{\Delta_{2}}{a_{2}} \exp (U)
\end{aligned}
$$

\section{$6 \tau$-function for the generalized DS hierarchy}

Now we will analyze the functional equations (16) for the case $N=2$. For convenience we present them here explicitly:

$$
\begin{aligned}
& \left(\frac{\mu-a_{1}}{\mu-b_{1}}\right) \chi_{11}(\lambda, \mu, \mathbf{x}+[\mathbf{a}])-\left(\frac{\lambda-a_{(1)}}{\lambda-b_{(1)}}\right) \chi_{11}(\lambda, \mu, \mathbf{x}+[\mathbf{b}])+ \\
& \left(b_{(1)}-a_{(1)}\right) \chi_{11}\left(\lambda, b_{(1)}, \mathbf{x}+[\mathbf{a}]\right) \chi_{11}\left(b_{(1)}, \mu, \mathbf{x}+[\mathbf{b}]\right)+ \\
& \left(b_{(2)}-a_{(2)}\right) \chi_{21}\left(\lambda, b_{(2)}, \mathbf{x}+[\mathbf{a}]\right) \chi_{12}\left(b_{(2)}, \mu, \mathbf{x}+[\mathbf{b}]\right)=0, \\
& \left(\frac{\mu-a_{(2)}}{\mu-b_{(2)}}\right) \chi_{22}(\lambda, \mu, \mathbf{x}+[\mathbf{a}])-\left(\frac{\lambda-a_{(2)}}{\lambda-b_{(2)}}\right) \chi_{22}(\lambda, \mu, \mathbf{x}+[\mathbf{b}])+ \\
& \left(b_{(1)}-a_{(1)}\right) \chi_{12}\left(\lambda, b_{(1)}, \mathbf{x}+[\mathbf{a}]\right) \chi_{21}\left(b_{(1)}, \mu, \mathbf{x}+[\mathbf{b}]\right)+ \\
& \left(b_{(2)}-a_{(2)}\right) \chi_{22}\left(\lambda, b_{(2)}, \mathbf{x}+[\mathbf{a}]\right) \chi_{22}\left(b_{(2)}, \mu, \mathbf{x}+[\mathbf{b}]\right)=0,
\end{aligned}
$$




$$
\begin{aligned}
& \left(\frac{\mu-a_{(1)}}{\mu-b_{(1)}}\right) \chi_{12}(\lambda, \mu, \mathbf{x}+[\mathbf{a}])-\left(\frac{\lambda-a_{(2)}}{\lambda-b_{(2)}}\right) \chi_{12}(\lambda, \mu, \mathbf{x}+[\mathbf{b}])+ \\
& \left(b_{(1)}-a_{(1)}\right) \chi_{12}\left(\lambda, b_{(1)}, \mathbf{x}+[\mathbf{a}]\right) \chi_{11}\left(b_{(1)}, \mu, \mathbf{x}+[\mathbf{b}]\right)+ \\
& \left(b_{(2)}-a_{(2)}\right) \chi_{22}\left(\lambda, b_{(2)}, \mathbf{x}+[\mathbf{a}]\right) \chi_{12}\left(b_{(2)}, \mu, \mathbf{x}+[\mathbf{b}]\right)=0, \\
& \left(\frac{\mu-a_{(2)}}{\mu-b_{(2)}}\right) \chi_{21}(\lambda, \mu, \mathbf{x}+[\mathbf{a}])-\left(\frac{\lambda-a_{(1)}}{\lambda-b_{(1)}}\right) \chi_{21}(\lambda, \mu, \mathbf{x}+[\mathbf{b}])+ \\
& \left(b_{(1)}-a_{(1)}\right) \chi_{11}\left(\lambda, b_{(1)}, \mathbf{x}+[\mathbf{a}]\right) \chi_{21}\left(b_{(1)}, \mu, \mathbf{x}+[\mathbf{b}]\right)+ \\
& \left(b_{(2)}-a_{(2)}\right) \chi_{21}\left(\lambda, b_{(2)}, \mathbf{x}+[\mathbf{a}]\right) \chi_{22}\left(b_{(2)}, \mu, \mathbf{x}+[\mathbf{b}]\right)=0 .
\end{aligned}
$$

First it is clear that equations (58) with $a_{2}=b_{2}$ and (59) with $a_{1}=b_{1}$ which coincide with those for scalar KP hierarchy give rise to the following expressions for $\chi_{11}$ and $\chi_{22}$ (see [12])

$$
\begin{aligned}
& \chi_{11}(\lambda, \mu, \mathbf{x})=\frac{1}{(\lambda-\mu)} \frac{\tau_{1}\left(\mathbf{x}_{\mathbf{1}}-[\lambda]+[\mu], \mathbf{x}_{\mathbf{2}}\right)}{\tau_{1}(\mathbf{x})}, \\
& \chi_{22}(\lambda, \mu, \mathbf{x})=\frac{1}{(\lambda-\mu)} \frac{\tau_{2}\left(\mathbf{x}_{\mathbf{1}}, \mathbf{x}_{\mathbf{2}}-[\lambda]+[\mu]\right)}{\tau_{2}(\mathbf{x})},
\end{aligned}
$$

where $\tau_{1}$ and $\tau_{2}$ are the components of the $\tau$-function which depend on the variables $\mathbf{x}=\left(\mathbf{x}_{1}, \mathbf{x}_{2}\right)$.

Let us consider now equation (59) at $\mu=a_{1}$ and $a_{2}=b_{2}$. Substituting the expression (62) into this equation, we obtain

$$
\begin{array}{r}
\tau_{1}\left(\mathbf{x}_{\mathbf{1}}+\left[b_{1}\right], \mathbf{x}_{\mathbf{2}}+\left[a_{2}\right]\right) \chi_{12}\left(\lambda, a_{1}, \mathbf{x}_{\mathbf{1}}+\left[b_{1}\right], \mathbf{x}_{\mathbf{2}}+\left[a_{2}\right]\right)= \\
\tau_{1}\left(\mathbf{x}_{\mathbf{1}}+\left[a_{1}\right], \mathbf{x}_{\mathbf{2}}+\left[a_{2}\right]\right) \chi_{12}\left(\lambda, a_{1}, \mathbf{x}_{\mathbf{1}}+\left[a_{1}\right], \mathbf{x}_{\mathbf{2}}+\left[a_{2}\right]\right) .
\end{array}
$$

For $b_{1}=0$ after the change $\mathbf{x}_{\mathbf{2}}+\left[a_{2}\right] \rightarrow \mathbf{x}_{\mathbf{2}}$, one gets

$$
\tau_{1}\left(\mathbf{x}_{\mathbf{1}}, \mathbf{x}_{\mathbf{2}}\right) \chi_{12}\left(\lambda, a_{1}, \mathbf{x}_{\mathbf{1}}, \mathbf{x}_{\mathbf{2}}\right)=\tau_{1}\left(\mathbf{x}_{\mathbf{1}}+\left[a_{1}\right], \mathbf{x}_{\mathbf{2}}\right) \chi_{12}\left(\lambda, 0, \mathbf{x}_{\mathbf{1}}+\left[a_{1}\right], \mathbf{x}_{\mathbf{2}}\right) .
$$

Denoting the r.h.s. of this equation as $\tilde{\tau}_{12}\left(\lambda, \mathbf{x}_{\mathbf{1}}+\left[a_{1}\right], \mathbf{x}_{\mathbf{2}}\right)$, we rewrite it as

$$
\chi_{12}\left(\lambda, \mu, \mathbf{x}_{\mathbf{1}}, \mathbf{x}_{\mathbf{2}}\right)=\frac{\tilde{\tau}_{12}\left(\lambda, \mathbf{x}_{\mathbf{1}}+[\mu], \mathbf{x}_{\mathbf{2}}\right)}{\tau_{1}\left(\mathbf{x}_{\mathbf{1}}, \mathbf{x}_{\mathbf{2}}\right)} .
$$

Then the substitution of (66) into equation (64) with $\mu=a_{1}$ and $\lambda=a_{2}$ gives rise to

$$
\begin{array}{r}
\tau_{1}\left(\mathbf{x}_{\mathbf{1}}+\left[a_{1}\right], \mathbf{x}_{\mathbf{2}}+\left[b_{2}\right]\right) \frac{\tilde{\tau}_{12}\left(a_{2}, \mathbf{x}_{\mathbf{1}}+\left[a_{1}\right]+\left[b_{1}\right], \mathbf{x}_{\mathbf{2}}+\left[a_{2}\right]\right)}{\tau_{1}\left(\mathbf{x}_{\mathbf{1}}+\left[a_{1}\right], \mathbf{x}_{\mathbf{2}}+\left[a_{2}\right]\right)}= \\
\tau_{2}\left(\mathbf{x}_{\mathbf{1}}+\left[a_{1}\right], \mathbf{x}_{\mathbf{2}}+\left[b_{2}\right]\right) \frac{\tilde{\tau}_{12}\left(b_{2}, \mathbf{x}_{\mathbf{1}}+\left[a_{1}\right]+\left[b_{1}\right], \mathbf{x}_{\mathbf{2}}+\left[b_{2}\right]\right)}{\tau_{1}\left(\mathbf{x}_{\mathbf{1}}+\left[a_{1}\right], \mathbf{x}_{\mathbf{2}}+\left[a_{2}\right]\right)} .
\end{array}
$$


This equation implies that

$$
\tilde{\tau}_{12}\left(a, \mathbf{x}_{\mathbf{1}}, \mathbf{x}_{\mathbf{2}}\right)=\frac{\tau_{12}\left(\mathbf{x}_{\mathbf{1}}, \mathbf{x}_{\mathbf{2}}-[a]\right)}{\rho\left(\mathbf{x}_{\mathbf{1}}, \mathbf{x}_{\mathbf{2}}\right)}
$$

and

$$
\rho\left(\mathbf{x}_{\mathbf{1}}, \mathbf{x}_{\mathbf{2}}\right)=\frac{\tau_{2}\left(\mathbf{x}_{\mathbf{1}}, \mathbf{x}_{\mathbf{2}}\right)}{\tau_{1}\left(\mathbf{x}_{\mathbf{1}}, \mathbf{x}_{\mathbf{2}}\right)} .
$$

where $\tau_{12}$ is an arbitrary function. Substitution of (69) into (66) gives

$$
\chi_{12}\left(\lambda, \mu, \mathbf{x}_{\mathbf{1}}, \mathbf{x}_{\mathbf{2}}\right)=\frac{\tau_{12}\left(\lambda, \mathbf{x}_{\mathbf{1}}+[\mu], \mathbf{x}_{\mathbf{2}}-[\lambda]\right)}{\tau_{2}\left(\mathbf{x}_{\mathbf{1}}, \mathbf{x}_{\mathbf{2}}\right)} .
$$

Now we proceed with equation (61). Substitution of the expression (63) into this equation with $\mu=a_{2}, a_{1}=b_{1}$ gives

$$
\begin{aligned}
& \chi_{21}\left(\lambda, a_{2}, \mathbf{x}_{\mathbf{1}}+\left[a_{1}\right], \mathbf{x}_{\mathbf{2}}+\left[b_{2}\right]\right)= \\
& \frac{\tau_{2}\left(\mathbf{x}_{\mathbf{1}}+\left[a_{1}\right], \mathbf{x}_{\mathbf{2}}+\left[a_{2}\right]\right)}{\tau_{2}\left(\mathbf{x}_{\mathbf{1}}+\left[a_{1}\right], \mathbf{x}_{\mathbf{2}}+\left[b_{2}\right]\right)} \chi_{21}\left(\lambda, b_{2}, \mathbf{x}_{\mathbf{1}}+\left[a_{1}\right], \mathbf{x}_{\mathbf{2}}+\left[a_{2}\right]\right)
\end{aligned}
$$

After the change $\mathbf{x}_{\mathbf{1}}+\left[a_{1}\right] \rightarrow \mathbf{x}_{\mathbf{1}}$ and the choice $b_{2}=0$ one gets

$$
\tau_{2}(\mathbf{x}) \chi_{21}\left(\lambda, a_{2}, \mathbf{x}\right)=\tau_{2}\left(\mathbf{x}_{\mathbf{1}}, \mathbf{x}_{\mathbf{2}}+\left[a_{2}\right]\right) \chi_{21}\left(\lambda, 0, \mathbf{x}_{\mathbf{1}}, \mathbf{x}_{\mathbf{2}}+\left[a_{2}\right]\right) .
$$

From this equation one concludes that

$$
\chi_{21}(\lambda, \mu, \mathbf{x})=\frac{\tilde{\tau}_{21}\left(\lambda, \mathbf{x}_{1}, \mathbf{x}_{2}+[\mu]\right)}{\tau_{2}(\mathbf{x})},
$$

where $\tilde{\tau}_{21}\left(\lambda, \mathbf{x}_{\mathbf{1}}, \mathbf{x}_{\mathbf{2}}\right)=\tau_{2}(\mathbf{x}) \chi_{21}(\lambda, 0, \mathbf{x})$.

Substituting now with $\mu=a_{2}, \lambda=a_{1}, b_{2}=0$ we obtain

$$
\frac{\tau_{1}\left(\mathbf{x}_{\mathbf{1}}+\left[a_{1}\right], \mathbf{x}_{\mathbf{2}}\right)}{\tau_{2}\left(\mathbf{x}_{\mathbf{1}}+\left[a_{1}\right], \mathbf{x}_{\mathbf{2}}\right)} \tilde{\tau}_{21}\left(a_{1}, \mathbf{x}_{\mathbf{1}}+\left[a_{1}\right], \mathbf{x}_{\mathbf{2}}\right)=\frac{\tau_{1}\left(\mathbf{x}_{\mathbf{1}}+\left[b_{1}\right], \mathbf{x}_{\mathbf{2}}\right)}{\tau_{2}\left(\mathbf{x}_{\mathbf{1}}+\left[b_{1}\right], \mathbf{x}_{\mathbf{2}}\right)} \tilde{\tau}_{21}\left(b_{1}, \mathbf{x}_{\mathbf{1}}+\left[b_{1}\right], \mathbf{x}_{\mathbf{2}}\right) .
$$

Hence

$$
\tilde{\tau}_{21}\left(a_{1}, \mathbf{x}_{\mathbf{1}}, \mathbf{x}_{\mathbf{2}}\right)=\frac{\tau_{21}\left(\mathbf{x}_{\mathbf{1}}-\left[a_{1}\right], \mathbf{x}_{\mathbf{2}}\right)}{\tilde{\rho}(\mathbf{x})}
$$

and

$$
\tilde{\rho}(\mathbf{x})=\frac{\tau_{1}(\mathbf{x})}{\tau_{2}(\mathbf{x})} .
$$


Substitution of (76) and (77) into (74) gives

$$
\chi_{21}(\lambda, \mu, \mathbf{x})=\frac{\tau_{21}\left(\mathbf{x}_{1}-[\lambda], \mathbf{x}_{2}+[\mu]\right)}{\tau_{1}(\mathbf{x})} .
$$

To establish the relation between $\tau_{1}$ and $\tau_{2}$ we consider again equation (75). Due to (76) one gets

$$
\frac{\tau_{1}\left(\mathbf{x}_{\mathbf{1}}+\left[b_{1}\right], \mathbf{x}_{\mathbf{2}}\right)}{\tau_{2}\left(\mathbf{x}_{\mathbf{1}}+\left[b_{1}\right], \mathbf{x}_{\mathbf{2}}\right)}=\frac{\tau_{1}\left(\mathbf{x}_{\mathbf{1}}+\left[a_{1}\right], \mathbf{x}_{\mathbf{2}}\right)}{\tau_{2}\left(\mathbf{x}_{\mathbf{1}}+\left[a_{1}\right], \mathbf{x}_{\mathbf{2}}\right)} .
$$

Since $a_{1}$ and $b_{1}$ are arbitrary parameters, one concludes that

$$
\frac{\tau_{1}(\mathbf{x})}{\tau_{2}(\mathbf{x})}=f\left(\mathbf{x}_{2}\right) .
$$

In the same way, it follows from (60) that

$$
\frac{\tau_{1}(\mathbf{x})}{\tau_{2}(\mathbf{x})}=f\left(\mathbf{x}_{1}\right) .
$$

Thus

$$
\frac{\tau_{1}(\mathbf{x})}{\tau_{2}(\mathbf{x})}=\text { const. }
$$

Having in mind the formulae (62) and (63), one can put $\tau_{1}=\tau_{2}$ without the loss of generality.

So for the 2-component KP hierarchy one has

$$
\begin{gathered}
\chi_{11}(\lambda, \mu, \mathbf{x})=\frac{1}{(\lambda-\mu)} \frac{\tau\left(\mathbf{x}_{1}-[\lambda]+[\mu], \mathbf{x}_{2}\right)}{\tau(\mathbf{x})}, \\
\chi_{22}(\lambda, \mu, \mathbf{x})=\frac{1}{(\lambda-\mu)} \frac{\tau\left(\mathbf{x}_{1}, \mathbf{x}_{\mathbf{2}}-[\lambda]+[\mu]\right)}{\tau(\mathbf{x})}, \\
\chi_{12}(\lambda, \mu, \mathbf{x})=\frac{\tau_{12}\left(\mathbf{x}_{\mathbf{1}}+[\mu], \mathbf{x}_{\mathbf{2}}-[\lambda]\right)}{\tau(\mathbf{x})} \\
\chi_{21}(\lambda, \mu, \mathbf{x})=\frac{\tau_{21}\left(\mathbf{x}_{1}-[\lambda], \mathbf{x}_{\mathbf{2}}+[\mu]\right)}{\tau(\mathbf{x})} .
\end{gathered}
$$

At this step $\tau, \tau_{12}$ and $\tau_{21}$ are arbitrary functions. Substitution of the expressions (83-86) into the general functional equations (58-61) gives rise to the four bilinear functional equations for these functions which represent themselves the addition formulae. We will write down them explicitly for the multicomponent case later. 


\section{$7 \quad \tau$-function and addition formulae for $\mathbf{n}$-component KP hierarchy}

It will be shown in the next section that the objects of multicomponent KP hierarchy depend not only on continuous times, but also on discrete times (indices). The addition formulae arising from the discrete dynamics will be considered in the context of Toda lattice hierarchy. Here we derive only the addition formulae following from the basic formula (16) defining the dynamics for continuous variables.

For the general multicomponent KP hierarchy the system (16) looks rather complicated. But its analysis is simplified considerably due to the special structure of equations (16). It isn't difficult to see that for two fixed values of $\alpha$ and $\beta$, taking $a_{\gamma}=b_{\gamma}, \gamma \neq \alpha, \gamma \neq \beta$, the system reduces to four equations of the DS hierarchy (58-61) for the variables $\mathbf{x}_{\alpha}$ and $\mathbf{x}_{\beta}$. Hence, in these variables we have the formulae

$$
\begin{aligned}
\chi_{\alpha \alpha}(\lambda, \mu, \mathbf{x}) & =\frac{1}{(\lambda-\mu)} \frac{\tau\left(\mathbf{x}_{\mathbf{1}}, \ldots, \mathbf{x}_{\alpha}-[\lambda]+[\mu], \ldots, \mathbf{x}_{\mathbf{N}}\right)}{\tau(\mathbf{x})} \\
\chi_{\alpha \beta}(\lambda, \mu, \mathbf{x}) & =\frac{\tau_{\alpha \beta}\left(\mathbf{x}_{\mathbf{1}}, \ldots, \mathbf{x}_{\alpha}+[\mu], \ldots, \mathbf{x}_{\beta}-[\lambda], \ldots, \mathbf{x}_{\mathbf{N}}\right)}{\tau(\mathbf{x})} \quad(\alpha \neq \beta) .(87)
\end{aligned}
$$

Here indices $\alpha$ and $\beta$ are arbitrary. Therefore the formulae (87) take place for all $\alpha$ and $\beta$.

To compactify these formulae we will introduce the shift operator $T_{\alpha}(\lambda)$ $(\alpha=1, \ldots, N)$

$$
T_{\alpha}(\lambda) f(\mathbf{x})=f\left(\mathbf{x}_{\mathbf{1}}, \ldots, \mathbf{x}_{\alpha}+[\lambda], \ldots, \mathbf{x}_{\mathbf{N}}\right) .
$$

Using such operators, one can present the formulae (87) in the form

$$
\begin{array}{r}
\chi_{\alpha \alpha}(\lambda, \mu, \mathbf{x})=\frac{1}{\lambda-\mu} \frac{T_{\alpha}(\mu) T_{\alpha}^{-1}(\lambda) \tau(\mathbf{x})}{\tau(\mathbf{x})} \quad \alpha=1, \ldots, N, \\
\chi_{\alpha \beta}(\lambda, \mu, \mathbf{x})=\frac{1}{\lambda-\mu} \frac{T_{\alpha}(\mu) T_{\beta}^{-1}(\lambda) \tau_{\alpha \beta}(\mathbf{x})}{\tau(\mathbf{x})} \quad \alpha \neq \beta .
\end{array}
$$

In the special cases $\lambda=0$ or $\mu=0$ these formulae reduce to the standard for-

mulae for the Baker-Akhiezer function [3], [14], [16]. Separating the diagonal case $\alpha=\beta$ and offdiagonal one, one gets

$$
\left(a_{\alpha}-\mu\right)\left(\lambda-b_{\alpha}\right) \tau(\mathbf{x}+[\mathbf{b}]) T_{\alpha}(\mu) T_{\alpha}^{-1}(\lambda) \tau(\mathbf{x}+[\mathbf{a}])+
$$




$$
\begin{aligned}
& \left(b_{\alpha}-\mu\right)\left(\lambda-a_{\alpha}\right) \tau(\mathbf{x}+[\mathbf{a}]) T_{\alpha}(\mu) T_{\alpha}^{-1}(\lambda) \tau(\mathbf{x}+[\mathbf{b}])+ \\
& \left(b_{\alpha}-a_{\alpha}\right)(\lambda-\mu) T_{\alpha}(\mu) T_{\alpha}^{-1}\left(b_{\alpha}\right) \tau(\mathbf{x}+[\mathbf{b}]) T_{\alpha}\left(b_{\alpha}\right) T_{\alpha}^{-1}(\lambda) \tau(\mathbf{x}+[\mathbf{a}])+ \\
& (\mu-\lambda)\left(\mu-b_{\alpha}\right)\left(\lambda-b_{\alpha}\right) \sum_{\gamma \neq \alpha}\left(b_{\gamma}-a_{\gamma}\right) T_{\alpha}(\mu) T_{\gamma}^{-1}\left(b_{\gamma}\right) \tau_{\alpha \gamma}(\mathbf{x}+[\mathbf{b}]) \times \\
& T_{\gamma}\left(b_{\gamma}\right) T_{\beta}^{-1}(\lambda) \tau_{\gamma \beta}(\mathbf{x}+[\mathbf{a}])=0
\end{aligned}
$$

and

$$
\begin{aligned}
& \left(\mu-a_{\alpha}\right)\left(\lambda-b_{\beta}\right) \tau(\mathbf{x}+[\mathbf{b}]) T_{\alpha}(\mu) T_{\beta}^{-1}(\lambda) \tau_{\alpha \beta}(\mathbf{x}+[\mathbf{a}])+ \\
& \left(\mu-b_{\alpha}\right)\left(\lambda-a_{\beta}\right) \tau(\mathbf{x}+[\mathbf{a}]) T_{\alpha}(\mu) T_{\beta}^{-1}(\lambda) \tau_{\alpha \beta}(\mathbf{x}+[\mathbf{b}])+ \\
& \left(b_{\alpha}-a_{\alpha}\right)\left(\lambda-b_{\beta}\right) T_{\alpha}(\mu) T_{\alpha}^{-1}\left(b_{\alpha}\right) \tau(\mathbf{x}+[\mathbf{b}]) T_{\alpha}\left(b_{\alpha}\right) T_{\beta}^{-1}(\lambda) \tau_{\alpha \beta}(\mathbf{x}+[\mathbf{a}])+ \\
& \left(\mu-b_{\alpha}\right)\left(\lambda-b_{\beta}\right) \sum_{\gamma \neq \alpha, \beta}\left(b_{\gamma}-a_{\gamma}\right) T_{\alpha}(\mu) T_{\gamma}^{-1}\left(b_{\gamma}\right) \tau_{\alpha \gamma}(\mathbf{x}+[\mathbf{b}]) \times \\
& T_{\gamma}\left(b_{\gamma}\right) T_{\beta}^{-1}(\lambda) \tau_{\gamma \beta}(\mathbf{x}+[\mathbf{a}])=0 .
\end{aligned}
$$

Equations (90) and (91) represent the extension of the simplest addition formula for the $\tau$-function in the scalar case (see [12]) to the multicomponent case. They are related to the generalization of the bilinear relations for the Plücker coordinates to the multicomponent case. On the other hand they could be connected with the possible multidimensional extensions of the Fay's trisecant formula [27].

More general addition formulae for the $\tau$-function follow from the general functional equation for the function $\chi_{\alpha \beta}$ which corresponds to the choice

$$
\left(g_{1}(\mathbf{x}) g_{2}^{-1}(\mathbf{x})\right)_{\alpha \beta}=\delta_{\alpha \beta} \prod_{k=1}^{N} \frac{\nu-a_{\alpha k}}{\nu-b_{\alpha k}} .
$$

\section{$8 \tau$-functional substitution and closed 1-form}

The formulae (83), (84), (85), (86) indicate that it is possible to preserve in multicomponent case the general formula connecting the $\tau$-function and the CBA function in the scalar case. It would be more consistent first to define the $\tau$-function through the CBA function in terms of the closed one-form, and then to prove that this definition implies the general formula. On the other hand, one may treat the general formula as the definition and then prove the

correctness of this definition reducing it to closed 1-form and showing the 
equivalence of 'local' and 'global' formulae (that implies unique existence of the $\tau$-function, at least in some neighborhood of $g=1$ ). In this paper we would rather follow the second way.

So we define the $\tau$-function by the relation

$$
\chi(\lambda, \mu, g)=\chi_{0}(\lambda, \mu) \frac{\tau\left(g_{(\lambda, \mu)} g\right)}{\tau(g)} .
$$

Here $\chi_{0}(\lambda, \mu)$ is just the function $(\lambda-\mu)^{-1}$ extended as 1 for $\lambda, \mu$ in different $D_{i}$, and the function $g_{(\lambda, \mu)}$ is an extension of the function $\frac{\nu-\lambda}{\nu-\mu}$ to the case of disconnected $G$

$$
\chi_{0}(\lambda, \mu)=\left\{\begin{array}{cl}
\frac{1}{\lambda-\mu} & \text { for } \lambda \in D_{i}, \mu \in D_{i} \\
1 & \text { for } \lambda \in D_{i}, \mu \in D_{j}, i \neq j .
\end{array}\right.
$$

The function $g_{(\lambda, \mu)}$ ( in fact, it is a set of $N$ loop group elements $g_{i}(\nu)$ depending on the parameters $\lambda, \mu)$ is the ratio of two terms

$$
\begin{aligned}
& g_{(\lambda, \mu)}=\frac{g_{(\lambda)}}{g_{(\mu)}}, \\
& g_{(\lambda) i}(\nu)=\left\{\begin{array}{cc}
\nu-\lambda & \text { for } \lambda \in D_{i} \\
1 & \text { for } \lambda \notin D_{i} .
\end{array}\right.
\end{aligned}
$$

To extract the formulae (83), (84), (85), (86) from the formula (93), one should recall that the loop group defining the multicomponent KP hierarchy can be decomposed in the following way

$$
g_{i}(\nu)=g_{i \text { out }}(\nu) \times \nu^{n_{i}}, \quad \sum_{i=1}^{N} n_{i}=0,
$$

where the first term defines the dependence on continuous times and the second - on the discrete times (indices). If $\lambda, \mu$ belong to the same $D_{i}$, using explicit parametrization of the loop group elements, we obtain exactly the formulae (83), (84). For $\lambda \in D_{k}, \mu \in D_{p}, k \neq p, g_{(\lambda, \mu)}$ has a factorization

$$
\begin{aligned}
& g_{(\lambda, \mu) k}(\nu)=\frac{\nu-\lambda}{\nu} \times \nu, \\
& g_{(\lambda, \mu) p}(\nu)=\frac{\nu}{\nu-\mu} \times \nu^{-1}, \\
& g_{(\lambda, \mu) i}(\nu)=1, \quad i \neq k, i \neq p .
\end{aligned}
$$


Using these formulae, we obtain the formulae (85), (86), with the components of $\tau$-function interpreted as a $\tau$-function with shifted index variables (general index zero is preserved). For example, in the two-component case the function $\tau_{12}$ corresponds to $\tau\left(n_{1}-1, n_{2}+1\right)$. To express the CBA function with zero indices, we need to know the set of functions $\tau, \tau_{i j}, i \neq j$. Then for other admissible index vectors the CBA function and the $\tau$-function can be found explicitly through the determinant formulae [13]. Thus, the discrete dynamics is exactly solvable in terms of initial data (CBA function with zero indices or the set of $\tau$-functions $\tau, \tau_{i j}, i \neq j$ ).

Returning to our original purpose, we will extract the definition of the $\tau$-function in terms of the closed 1-form from (2) and then prove that both definitions are equivalent. Our derivation will mostly repeat the derivation in the scalar case given in [12], but we would like to remind it due to the importance for the following consideration.

Differentiating (93) with respect to $\lambda$, one obtains

$$
-\frac{1}{\tau(g)} \oint \frac{\delta \tau\left(g \times g_{(\lambda, \mu)}\right)}{\delta \ln g_{\alpha}(\nu)} \frac{1}{\nu-\lambda} d \nu=\frac{\partial}{\partial \lambda}(\lambda-\mu) \chi_{\alpha \alpha}(\lambda, \mu ; g),
$$

or for $\lambda=\mu$

$$
-\frac{1}{\tau(g)} \oint \frac{\delta \tau(g)}{\delta \ln g_{\alpha}(\nu)} \frac{1}{\nu-\lambda} d \nu=\chi_{\mathrm{r} \alpha \alpha}(\lambda, \lambda ; g)
$$

where $\chi_{\mathrm{r} \alpha \beta}(\lambda, \mu ; g)=\chi_{\alpha \beta}(\lambda, \mu ; g)-\delta_{\alpha \beta}(\lambda-\mu)^{-1}$,'r' is for 'regularized'. This equation can be rewritten in the form

$$
-\frac{1}{\tau(g)} \oint \frac{\delta \tau(g)}{\delta \ln g_{\alpha}(\nu)} \frac{1}{\nu-\lambda} d \nu=\oint \chi_{\mathrm{r} \alpha \alpha}(\nu, \nu ; g) \frac{1}{\nu-\lambda} d \nu .
$$

Relation (100) implies that the linear functionals $-\frac{1}{\tau(g)} \frac{\delta \tau(g(\nu))}{\delta \ln g}$ and $\chi_{\mathrm{r}}(\nu, \nu ; g)$ are identical for arbitrary variations $\frac{\delta g}{g}$ analytic outside each $D_{i}$ and decreasing at infinity. Thus for $\frac{\delta g}{g}$ belonging to this class of functions the variation of the $\tau$-function is given by

$$
-\delta \ln \tau(g)=\sum_{\alpha=1}^{N} \oint \chi_{\mathrm{r} \alpha \alpha}(\nu, \nu ; g) \frac{\delta g_{\alpha}(\nu)}{g_{\alpha}(\nu)} d \nu .
$$


This expression defines a variational 1-form defining the $\tau$-function. It is easy to prove using the identity (11) that this form is closed. Indeed, according to (11) (here we are using scalar notations, it is not difficult to extract equations in terms of components $\left.\chi_{\alpha \beta}(\lambda, \mu ; g)\right)$

$$
\begin{gathered}
\delta \chi(\lambda, \mu ; g)=\int_{\partial G} \chi(\nu, \mu ; g) \frac{\delta g(\nu)}{g(\nu)} \chi(\lambda, \nu ; g) d \nu, \\
\delta \chi_{\mathrm{r}}(\lambda, \lambda ; g)=\int_{\partial G} \chi_{r}(\nu, \lambda ; g) \frac{\delta g(\nu)}{g(\nu)} \chi_{\mathrm{r}}(\lambda, \nu ; g) d \nu .
\end{gathered}
$$

So the variation of the (101) gives

$$
-\delta^{2} \ln \tau(g)=\int_{\partial \mathcal{G}} \int_{\partial G} \chi_{\mathrm{r}}(\nu, \lambda ; g) \chi_{\mathrm{r}}(\lambda, \nu ; g) \frac{\delta^{\prime} g(\nu)}{g(\nu)} \frac{\delta g(\lambda)}{g(\lambda)} d \nu d \lambda .
$$

The symmetry of the kernel of second variation with respect to $\lambda, \nu$ implies that the form (101) is closed.

So the formula (101) gives the definition of the $\tau$-function in terms of the closed 1-form. For the standard KP coordinates

$$
\frac{\delta g_{\alpha}(\lambda)}{g_{\alpha}(\lambda)}=\sum_{n=1}^{\infty} \frac{d x_{(\alpha) n}}{\lambda^{n}}
$$

This formula allows us to obtain a closed 1-form in terms of $d x_{\alpha n}$

$$
-d \ln \tau(\mathbf{k}, \mathbf{x})=\left.\sum_{\alpha=1}^{N} \sum_{n=0}^{\infty} \frac{\partial^{n}}{\partial \nu^{n}} \chi_{\mathrm{r} \alpha \alpha}(\nu, \nu, \mathbf{k}, \mathbf{x})\right|_{\nu=0} d x_{(\alpha) n} .
$$

To prove that the 'local' definition in terms of the closed 1-form implies the global definition (93), it is sufficient to show that logarithmic derivatives of the l.h.s. and the r.h.s. of (93) with respect to $\lambda, \mu$ and variational logarithmic derivatives with respect to $\delta g_{\alpha} / g_{\alpha}$ are equal in virtue of (101)

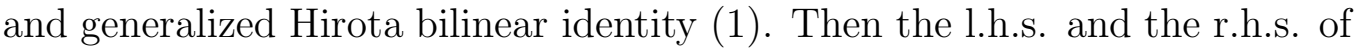
(93) could differ only by the factor, and the normalization of the function $\chi$ implies that this factor is equal to 1.

Here we will prove only the equality of logarithmic variations, the proof for derivatives is analogous (see [12]). 
The logarithmic variation of (93) gives

$$
\begin{aligned}
& \delta\left(\ln \chi(\lambda, \mu ; g)(\lambda-\mu)-\ln \tau\left(g \times g_{(\lambda, \mu)}\right)+\ln \tau(g)\right)= \\
& \frac{1}{\chi(\lambda, \mu)} \int_{\partial G} \chi(\nu, \mu ; g) \frac{\delta g(\nu)}{g(\nu)} \chi(\lambda, \nu ; g) d \nu \\
& -\int_{\partial G}\left(\chi_{\mathrm{r}}\left(\nu, \nu ; g \times g_{(\lambda, \mu)}\right)-\chi_{\mathrm{r}}(\nu, \nu ; g)\right) \frac{\delta g(\nu)}{g(\nu)} d \nu
\end{aligned}
$$

(we have used (102) and (101)). After the use of the determinant formula [13]

$$
\chi\left(\lambda, \mu ; g \times g_{\left(\lambda^{\prime}, \mu^{\prime}\right)}\right)=g_{\left(\lambda^{\prime}, \mu^{\prime}\right)}^{-1}(\lambda) g_{\left(\lambda^{\prime}, \mu^{\prime}\right)}(\mu) \frac{\left|\begin{array}{cc}
\chi\left(\lambda^{\prime}, \mu^{\prime}\right) & \chi\left(\lambda^{\prime}, \mu\right) \\
\chi\left(\lambda, \mu^{\prime}\right) & \chi(\lambda, \mu)
\end{array}\right|}{\chi\left(\lambda^{\prime}, \mu^{\prime}\right)}
$$

to transform the second term, one concludes that the variation is equal to zero.

\section{Generalized 2D Toda lattice hierarchy. Ba- sic equations.}

We will consider here for simplicity only scalar 2D Toda lattice hierarchy. It is generated by the generalized Hirota identity with the domain $G$ which consists of two disconnected regions, i.e. in some sense it is a two-point KP hierarchy. But it also possess some fundamental special features and can be treated as a primary object with respect to scalar KP hierarchy.

Without loss of generality one can choose $G$ as

$$
G=D_{0} \cup D_{\infty}
$$

where $D_{0}$ and $D_{\infty}$ are unit discs around the origin and infinity respectively. The two-component KP hierarchy is generated by the pair of functions $g_{\text {in }}$, $g_{\text {out }}$ defined on the boundary of $D_{0}, D_{\infty}$ respectively, having zero general index (let their individual indices be $n,-n), g_{\text {out }}$ is analytic and have no zeroes outside the unit circle, $g_{\text {in }}$ inside the unit circle. It is easy to see, that 
dynamics defined by the pair of these functions coincides with the dynamics defined by one function for both boundaries

$$
g=\lambda^{n} g_{\text {in }} g_{\text {out }}
$$

up to some trivial dynamics, defined by a function analytic in $G=D_{0} \cup D_{\infty}$ $\left(g=g_{\text {in }}, \lambda \in D_{0} ; g=g_{\text {out }}, \lambda \in D_{\infty}\right)$.

As we know, arbitrary function on the circle having no zeroes (arbitrary loop) can be represented in the form (107). So in the case of Toda lattice hierarchy the objects of the theory nontrivially depend on the whole loop group (i.e. on discrete time, positive and negative continuous times), $\tau$ function in this case is a functional of arbitrary $g$ on the circle. Discrete dynamics is correctly defined, because in this case $\lambda^{n}$ is a function having in $G$ equal number of zeroes and poles.

The explicit parametrization of the loop group element $g$ in terms of times is given by

$$
g(n, \mathbf{x}, \nu)=g\left(n, \mathbf{x}_{+}, \mathbf{x}_{-}, \nu\right)=\nu^{n} \exp \left(\sum_{k=1}^{\infty} x_{(+) k} \nu^{-k}+x_{(-) k} \nu^{k}\right) .
$$

The compact form of 2D Toda lattice hierarchy in terms of the function $\chi(\lambda, \mu, k, \mathbf{x})$ can be obtained from (1) using special combinations of group elements $g_{1} g_{2}^{-1}$ with simple analytical properties. To define dynamics with respect to continuous times, let us take

$$
\begin{aligned}
& g_{0}(\nu):=g_{1}(\nu) g_{2}^{-1}(\nu)=\frac{\nu-a_{+}}{\nu-b_{+}} \frac{\nu-a_{-}}{\nu-b_{-}}, \\
& a_{+}, b_{+} \in D_{0}, a_{-}, b_{-} \in D_{\infty}, \\
& g_{0}(\nu)=g_{+} \times\left(g_{-}\right)^{-1}, \quad g_{+}=\frac{\nu-a_{+}}{\nu-b_{+}}, \quad g_{-}=\frac{\nu-b_{-}}{\nu-a_{-}} .
\end{aligned}
$$

Substituting the expression for $g_{1} g_{2}^{-1}$ into the generalized Hirota bilinear identity (11), one obtains

$$
\begin{aligned}
& g_{0}(\mu) \chi\left(\lambda, \mu, g \times g_{+}\right)-g_{0}(\lambda) \chi\left(\lambda, \mu, g \times g_{-}\right)+ \\
& \left.\operatorname{Res}\left(g_{0}\right)\right|_{b_{+}} \chi\left(\lambda, b_{+}, g \times g_{+}\right) \chi\left(b_{+}, \mu, g \times g_{-}\right)+ \\
& \left.\operatorname{Res}\left(g_{0}\right)\right|_{b_{-}} \chi\left(\lambda, b_{-}, g \times g_{+}\right) \chi\left(b_{-}, \mu, g \times g_{-}\right)=0 .
\end{aligned}
$$


The equations of Toda lattice hierarchy are generated by equation (110) with $b_{+}=0, b_{-}=\infty$, or, in terms of $g_{0}$

$$
\begin{array}{r}
g_{0}(\nu)=g_{1}(\nu) g_{2}^{-1}(\nu)=\left(1-\frac{\nu}{a_{-}}\right)\left(1-\frac{a_{+}}{\nu}\right)=\exp \left(\sum_{i=1}^{\infty} \frac{1}{i}\left(\left(\frac{\nu}{a_{-}}\right)^{i}+\left(\frac{a_{+}}{\nu}\right)^{i}\right)\right)(111 . \\
\left|a_{+}\right|<1,\left|a_{-}\right|>1 .
\end{array}
$$

Substituting into equation (110) parametrization of loop group elements in terms of times, we obtain

$$
\begin{aligned}
& \left(1-\frac{\mu}{a_{-}}\right)\left(1-\frac{a_{+}}{\mu}\right) \chi\left(\lambda, \mu, n, \mathbf{x}_{+}+\left[\mathbf{a}_{+}\right], \mathbf{x}_{-}\right)- \\
& \left(1-\frac{\lambda}{a_{-}}\right)\left(1-\frac{a_{+}}{\lambda}\right) \chi\left(\lambda, \mu, n, \mathbf{x}_{+}, \mathbf{x}_{-}-\left[\mathbf{a}_{-}\right]\right)+ \\
& -a_{+} \chi\left(\lambda, 0, n, \mathbf{x}_{+}+\left[\mathbf{a}_{+}\right], \mathbf{x}_{-}\right) \chi\left(0, \mu, n, \mathbf{x}_{+}, \mathbf{x}_{-}-\left[\mathbf{a}_{-}\right]\right) \\
& -\frac{1}{a_{-}} \chi\left(\lambda, \infty, n, \mathbf{x}_{+}+\left[\mathbf{a}_{+}\right], \mathbf{x}_{-}\right) \chi\left(\infty, \mu, n, \mathbf{x}_{+}, \mathbf{x}_{-}-\left[\mathbf{a}_{-}\right]\right)=0
\end{aligned}
$$

where $\chi(\lambda, \underset{\sim}{\infty})=\chi(\lambda, \mu) \times\left.\mu\right|_{\mu=\infty}$. In terms of CBA and BA functions $\psi(\lambda, \mu)$ and $\psi_{0}(\lambda), \tilde{\psi}_{0}(\mu) \psi_{\infty}(\lambda), \tilde{\psi}_{\infty}(\mu)$ we have

$$
\begin{aligned}
& \psi\left(\lambda, \mu, n, \mathbf{x}_{+}+\left[\mathbf{a}_{+}\right], \mathbf{x}_{-}\right)-\psi\left(\lambda, \mu, n, \mathbf{x}_{+}, \mathbf{x}_{-}-\left[\mathbf{a}_{-}\right]\right)= \\
& a_{+} \psi_{0}\left(\lambda, n, \mathbf{x}_{+}+\left[\mathbf{a}_{+}\right], \mathbf{x}_{-}\right) \tilde{\psi}_{0}\left(\mu, n, \mathbf{x}_{+}, \mathbf{x}_{-}-\left[\mathbf{a}_{-}\right]\right)+ \\
& \frac{1}{a_{-}} \psi_{\infty}\left(\lambda, n, \mathbf{x}_{+}+\left[\mathbf{a}_{+}\right], \mathbf{x}_{-}\right) \tilde{\psi}_{\infty}\left(\mu, n, \mathbf{x}_{+}, \mathbf{x}_{-}-\left[\mathbf{a}_{-}\right]\right) .
\end{aligned}
$$

To get equations defining the discrete dynamics, we take

$$
g_{0}(\nu)=g_{1}(\nu) g_{2}^{-1}(\nu)=\frac{1}{\lambda}
$$

or

$$
g_{0}(\nu)=g_{1}(\nu) g_{2}^{-1}(\nu)=\lambda .
$$

In the first case the equation for the function $\chi$ reads

$$
\begin{aligned}
& \frac{1}{\mu} \chi\left(\lambda, \mu, n+1, \mathbf{x}_{+}, \mathbf{x}_{-}\right)-\frac{1}{\lambda} \chi\left(\lambda, \mu, n, \mathbf{x}_{+}, \mathbf{x}_{-}\right)= \\
& \chi\left(\lambda, 0, n+1, \mathbf{x}_{+}, \mathbf{x}_{-}\right) \chi\left(0, \mu, n, \mathbf{x}_{+}, \mathbf{x}_{-}\right),
\end{aligned}
$$


and in the second case

$$
\begin{aligned}
& \mu \chi\left(\lambda, \mu, n, \mathbf{x}_{+}, \mathbf{x}_{-}\right)-\lambda \chi\left(\lambda, \mu, n+1, \mathbf{x}_{+}, \mathbf{x}_{-}\right)= \\
& \chi\left(\lambda, \infty, n, \mathbf{x}_{+}, \mathbf{x}_{-}\right) \chi\left(\infty, \mu, n+1, \mathbf{x}_{+}, \mathbf{x}_{-}\right) .
\end{aligned}
$$

In terms of $\mathrm{CBA}$ and $\mathrm{BA}$ functions one gets

$$
\begin{aligned}
& \psi\left(\lambda, \mu, n+1, \mathbf{x}_{+}, \mathbf{x}_{-}\right)-\psi\left(\lambda, \mu, n, \mathbf{x}_{+}, \mathbf{x}_{-}\right)= \\
& \psi_{0}\left(\lambda, n+1, \mathbf{x}_{+}, \mathbf{x}_{-}\right) \tilde{\psi}_{0}\left(\mu, n, \mathbf{x}_{+}, \mathbf{x}_{-}\right) \\
& \psi\left(\lambda, \mu, n, \mathbf{x}_{+}, \mathbf{x}_{-}\right)-\psi\left(\lambda, \mu, n+1, \mathbf{x}_{+}, \mathbf{x}_{-}\right)= \\
& \psi_{\infty}\left(\lambda, n, \mathbf{x}_{+}, \mathbf{x}_{-}\right) \tilde{\psi}\left(\mu, n+1, \mathbf{x}_{+}, \mathbf{x}_{-}\right) .
\end{aligned}
$$

Equations (113), (118) define the generalized 2D Toda lattice hierarchy. The discrete dynamics can be resolved in both directions explicitly (i.e. $\chi(n+1)$, $\chi(n-1)$ can be expressed through $\chi(n))$ using the determinant formula [13].

\section{Toda lattice hierarchy: 2DTL, 2D Volterra chain, 2DTL singularity manifold equa- tion}

At this point we would like to derive the simplest equations of generalized 2D Toda lattice hierarchy. They contain three variables: one discrete variable $n$ and two continuous variables $x_{(+) 1}, x_{(-) 1}$ (we will denote them $x_{+}$and $x_{-}$). We will derive three different equation corresponding to different levels of the tower of equations of generalized hierarchy: the main equation (the standard two-dimensional Toda lattice equation), the modified equation (i.e. equation for Baker-Akhiezer function) and singularity manifold type equation (arising here as an equation for the CBA function). It is interesting to note that the second and the third equation (not to mention the first) are already known in literature in different contexts (see [19], [18], [20]).

The basic equations for our derivation are equations (116), (117) and the zero order terms of expansion of $(\underline{112})$ in $a_{+}, 1 / a_{-}$, i.e.

$$
\begin{aligned}
& \left(\frac{\partial}{\partial x_{+}}+\frac{1}{\lambda}-\frac{1}{\mu}\right) \chi\left(\lambda, \mu, n, \mathbf{x}_{+}, \mathbf{x}_{-}\right)= \\
& \chi\left(\lambda, 0, n, \mathbf{x}_{+}, \mathbf{x}_{-}\right) \chi\left(0, \mu, n, \mathbf{x}_{+}, \mathbf{x}_{-}\right)
\end{aligned}
$$




$$
\begin{aligned}
& \left(\frac{\partial}{\partial x_{-}}+\lambda-\mu\right) \chi\left(\lambda, \mu, n, \mathbf{x}_{+}, \mathbf{x}_{-}\right)= \\
& \chi\left(\lambda, \infty, n, \mathbf{x}_{+}, \mathbf{x}_{-}\right) \chi\left(\infty, \mu, n, \mathbf{x}_{+}, \mathbf{x}_{-}\right)
\end{aligned}
$$

In terms of CBA and BA functions instead of (119) we have

$$
\begin{aligned}
& \frac{\partial}{\partial x_{+}} \psi\left(\lambda, \mu, n, \mathbf{x}_{+}, \mathbf{x}_{-}\right)=\psi_{0}\left(\lambda, n, \mathbf{x}_{+}, \mathbf{x}_{-}\right) \tilde{\psi}_{0}\left(\mu, n, \mathbf{x}_{+}, \mathbf{x}_{-}\right) \\
& \frac{\partial}{\partial x_{-}} \psi\left(\lambda, \mu, n, \mathbf{x}_{+}, \mathbf{x}_{-}\right)=\psi_{\infty}\left(\lambda, n, \mathbf{x}_{+}, \mathbf{x}_{-}\right) \tilde{\psi}_{\infty}\left(\mu, n, \mathbf{x}_{+}, \mathbf{x}_{-}\right) .
\end{aligned}
$$

Combining (116), (117) and (119), one obtains

$$
\begin{aligned}
& \left(\frac{\partial}{\partial x_{+}}+\frac{1}{\lambda}\right) \chi\left(\lambda, \mu, n, \mathbf{x}_{+}, \mathbf{x}_{-}\right)-\frac{1}{\lambda} \chi\left(\lambda, \mu, n-1, \mathbf{x}_{+}, \mathbf{x}_{-}\right)= \\
& \chi\left(\lambda, 0, n, \mathbf{x}_{+}, \mathbf{x}_{-}\right)\left(\chi\left(0, \mu, n, \mathbf{x}_{+}, \mathbf{x}_{-}\right)-\chi\left(0, \mu, n-1, \mathbf{x}_{+}, \mathbf{x}_{-}\right)\right) \\
& \left(\frac{\partial}{\partial x_{-}}+\lambda\right) \chi\left(\lambda, \mu, n, \mathbf{x}_{+}, \mathbf{x}_{-}\right)-\lambda \chi\left(\lambda, \mu, n+1, \mathbf{x}_{+}, \mathbf{x}_{-}\right)= \\
& \chi\left(\lambda, \infty, n, \mathbf{x}_{+}, \mathbf{x}_{-}\right)\left(\chi\left(\infty, \mu, n, \mathbf{x}_{+}, \mathbf{x}_{-}\right)-\chi\left(\infty, \mu, n+1, \mathbf{x}_{+}, \mathbf{x}_{-}\right)\right)(12
\end{aligned}
$$

Taking the first equation at $\lambda=\infty, \mu=0$, the second at $\lambda=0, \mu=\infty$, one obtains the following equations

$$
\begin{aligned}
& \partial_{+} u(n)=(U(n)-U(n-1)) u(n), \\
& \partial_{-} v(n)=(V(n)-V(n+1)) v(n) .
\end{aligned}
$$

Here we have introduced the notations

$$
\begin{aligned}
& u(n)=\chi\left(\infty, 0, n, \mathbf{x}_{+}, \mathbf{x}_{-}\right) \\
& v(n)=\chi\left(0, \infty, n, \mathbf{x}_{+}, \mathbf{x}_{-}\right) \\
& U(n)=\chi_{\mathrm{r}}\left(0,0, n, \mathbf{x}_{+}, \mathbf{x}_{-}\right) \\
& V(n)=\chi_{\mathrm{r}}\left(\infty, \infty, n, \mathbf{x}_{+}, \mathbf{x}_{-}\right),
\end{aligned}
$$

and we omit arguments $\mathbf{x}_{+}, \mathbf{x}_{-}$common for all functions.

Equations (119) taken respectively at $\lambda=\infty, \mu=\infty ; \lambda=0, \mu=0$ give rise to the following relations

$$
\begin{aligned}
& \partial_{+} V(n)=u(n) v(n)-1, \\
& \partial_{-} U(n)=u(n) v(n)-1,
\end{aligned}
$$


which together with (122) form a closed system of equations.

There is also an additional relation implied by (116), (117):

$$
u(n+1) v(n)=1
$$

This formula shows that the functions $u(n)$ and $v(n)$ are not independent.

The system of equations (122), (123), (124) represent some special form of classical 2D Toda lattice equations. To show it, let us derive more standard forms of Toda lattice from this system. First, it is easy to show that

$$
\begin{aligned}
& \partial_{+} \partial_{-} \ln u(n)=u(n) v(n)-u(n-1) v(n-1), \\
& \partial_{+} \partial_{-} \ln v(n)=u(n) v(n)-u(n+1) v(n+1) .
\end{aligned}
$$

Thus for the function $h(n)=u(n) v(n)$ we have an equation

$$
\partial_{+} \partial_{-} \ln h(n)=2 h(n)-h(n-1)-h(n+1),
$$

which is $2 \mathrm{D}$ Toda lattice equation in the Darboux form.

Since, according to (124), the functions $u(n)$ and $v(n)$ are not independent, we can write an equation for one function

$$
\partial_{+} \partial_{-} \ln u(n)=\frac{u(n)}{u(n+1)}-\frac{u(n-1)}{u(n)} .
$$

In terms of $\theta(n)=\ln u(n)$ we have

$$
\partial_{+} \partial_{-} \theta(n)=\exp (\theta(n)-\theta(n+1))-\exp (\theta(n-1)-\theta(n)) .
$$

This is just a standard form of the 2D Toda lattice equation. An original bilinear form, introduced by Toda, follows from addition formulae for the $\tau$-function, which will be given in the next section.

Now we proceed to the modified Toda lattice equation. In this case we will use the equations for generic wave functions, which are given by (118) and (120) integrated over $\lambda$ and $\mu$ with some weight functions $\rho(\lambda)$ and $\eta(\mu)$ (in fact, each of them in matrix notations is a pair of functions defined on 'in' unit circle and 'out' unit circle, see also (31)):

$$
\begin{aligned}
& \Phi(n)-\Phi(n+1)=\Psi_{0}(n+1) \widetilde{\Psi}_{0}(n) \\
& \Phi(n)-\Phi(n-1)=\Psi_{\infty}(n-1) \widetilde{\Psi}_{\infty}(n)
\end{aligned}
$$


(we have omitted common arguments $\mathbf{x}_{+}, \mathbf{x}_{-}$)

Combining these equations, one obtains a linear problem for the modified Toda lattice equation

$$
\begin{aligned}
& \partial_{+} \Phi=W_{+}(1-T) \Phi, \quad W_{+}=\frac{\psi_{0}}{T \psi_{0}}, \\
& \partial_{-} \Phi=W_{-}\left(1-T^{-1}\right) \Phi, \quad W_{-}=\frac{\psi_{\infty}}{T^{-1} \psi_{\infty}} .
\end{aligned}
$$

The compatibility condition gives the modified Toda lattice equations

$$
\begin{aligned}
& \partial_{-} W_{+}=W_{+}(1-T) W_{-}, \\
& \partial_{+} W_{-}=W_{-}\left(1-T^{-1}\right) W_{+} .
\end{aligned}
$$

These equations quite recently appeared in literature [19], 18] under the name of two-dimensional Volterra chain. It is interesting to note that in 18] they arose in the geometrical context.

Proceeding further, one can obtain an equation for the function $\Phi$, using linear operators of modified Toda lattice to express the functions $W_{+}, W_{-}$ via the function $\Phi$ and then substituting the final result to equations (131). Both equations give rise to the same equation

$$
\partial_{+} \partial_{-} \Phi+\partial_{+} \Phi \cdot \partial_{-} \Phi\left(\frac{1}{T \Phi-\Phi}+\frac{1}{T^{-1} \Phi-\Phi}\right)=0
$$

This equation was also introduced in different contexts in [19], [18]. Another interesting feature of equation (132) could be its connection with the Painleve analysis of the 2DTL. Making comparison with one-dimensional Toda lattice case [20], and recalling analogous equation for the scalar KP case, it is natural to suggest that the equation (132) represent a singularity manifold equation for the 2DTL.

Equation (132) generates 2DTL and two-dimensional Volterra chain in different ways: first, in terms of Combescure invariants, and second, in terms of expansion in $\lambda, \mu$ of the function $\psi\left(\lambda, \mu, n, \mathbf{x}_{+}, \mathbf{x}_{-}\right)$which satisfies equation (132).

The Combescure invariants for (132) are given by the formulae

$$
\begin{aligned}
& W_{+}=-\frac{\partial_{+} \Phi}{\Delta \Phi} \\
& W_{-}=\frac{\partial_{-} T \Phi}{\Delta \Phi}
\end{aligned}
$$


(we have written down only 'left' invariants). For two-dimensional Volterra chain the Combescure invariants look like

$$
\begin{aligned}
& u=W_{-} \exp \left(-\Delta^{-1}\left(W_{-}+T W_{+}\right)\right), \\
& v=W_{+} \exp \left(\Delta^{-1}\left(W_{-}+T W_{+}\right)\right) .
\end{aligned}
$$

Substituting (133) to (134), one obtains expressions of the full Combescure group invariants for singularity manifold equation (132).

At last, we present the formulae for the hierarchies of two-dimensional Toda, Volterra and 2D Toda singularity manifold equations. The derivation is very similar to the case of DS hierarchy, so we will omit it.

The 2DTL hierarchy can be represented as an equation

$$
T_{+} T_{-}^{-1} u+\frac{a_{+}}{a_{-}} T u=T_{+} u T_{-}^{-1} u\left(\frac{1}{u}+\frac{a_{+}}{a_{-}} \frac{1}{T_{+} T_{-}^{-1} T^{-1} u}\right)
$$

where a convention for shift and difference operators is standard

$$
\begin{aligned}
& T_{+}: \mathbf{x}_{+} \rightarrow \mathbf{x}_{+}+\left[a_{+}\right], \\
& T_{-}: \mathbf{x}_{-} \rightarrow \mathbf{x}_{+}+\left[a_{-}^{-1}\right], \\
& \Delta_{+}=T_{+}-1, \\
& \Delta_{-}=\left(T_{-}-1\right) .
\end{aligned}
$$

The 2D Volterra hierarchy reads

$$
\begin{aligned}
& a_{-} \Delta_{-} W_{+}+\left(T_{-} W_{+}\right)\left(T W_{-}\right)-\left(T_{+} W_{-}\right) W_{+}=0, \\
& 1 / a_{+} \Delta_{+} W_{-}+\left(T_{+} W_{-}\right)\left(T^{-1} W_{+}\right)-\left(T_{-} W_{+}\right) W_{-}=0,
\end{aligned}
$$

where $W_{+}=\frac{T_{+} \Psi_{0}}{T \Psi_{0}}, W_{-}=\frac{T_{-} \Psi_{\infty}}{T^{-1} \Psi_{\infty}}$.

Finally, 2DTL singularity manifold equation hierarchy looks like

$$
\begin{aligned}
& \left\{T_{-}\left(T_{+}-1\right) \Phi\right\}\left\{T\left(T_{-}-T^{-1}\right) \Phi\right\}\left\{T_{+}\left(1-T^{-1}\right) \Phi\right\}= \\
& \left\{\left(T_{+}-1\right) \Phi\right\}\left\{T_{+}\left(T_{-}-T^{-1}\right) \Phi\right\}\left\{T_{-}(T-1) \Phi\right\} .
\end{aligned}
$$

This equation possesses a symmetry $T_{+} \rightarrow T_{-}, T_{-} \rightarrow T_{+}, T \rightarrow T^{-1}$. Though this symmetry is not obvious but it can be easily verified. 


\section{Addition formulae for Toda lattice hierar- chy}

The $\tau$-functional substitution

$$
\begin{array}{r}
\chi(\lambda, \mu, g)=\frac{1}{\lambda-\mu} \frac{\tau\left(g_{(\lambda, \mu)} g\right)}{\tau(g)}, \\
g_{(\lambda, \mu)}=\frac{\lambda-\nu}{\mu-\nu},
\end{array}
$$

to (110), (118) leads to addition formulae for the $\tau$-function, the expansion of which gives the equations of the Toda lattice hierarchy in terms of the $\tau$-function.

The simplest addition formulae follow from (118)

$$
\begin{aligned}
& \frac{1}{\mu(\lambda-\mu)} \tau\left(g \times g_{\mathrm{d}} g_{(\lambda, \mu)}\right) \tau(g)-\frac{1}{\lambda(\lambda-\mu)} \tau\left(g \times g_{(\lambda, \mu)}\right) \tau\left(g \times g_{d}\right)+ \\
& \frac{1}{\lambda \mu} \tau\left(g \times g_{\mathrm{d}} g_{(\lambda, 0)}\right) \tau\left(g \times g_{(0, \mu)}\right)=0 .
\end{aligned}
$$

This is a functional form of addition formulae, containing four components characterized by the position of $\lambda, \mu$ with respect to the unit circle. Using parametrization of loop group elements in terms of times, we obtain a standard form of addition formulae. It is convenient to write down first the factorization formulae for the function $g_{(\lambda, \mu)}$, representing it as a product of functions analytic inside and outside the unit circle for different positions of $\lambda$ and $\mu$. For $\lambda, \mu$ both belonging to $D_{0}\left(D_{\infty}\right)$ the factorization is trivial, because $g_{(\lambda, \mu)}$ is analytic outside (inside) the unit circle. For $\lambda, \mu$ in different domains it is given by

$$
\begin{aligned}
& g_{\left(\lambda_{+}, \mu_{-}\right)}=g_{\mathrm{d}} g_{\left(\lambda_{+}, 0\right)} g_{\left(\infty, \mu_{-}\right)}, \\
& g_{\left(\lambda_{-}, \mu_{+}\right)}=g_{\mathrm{d}}^{-1} g_{\left(\lambda_{-}, \infty\right)} g_{\left(0, \mu_{+}\right)}, \\
& g_{\mathrm{d}}(\nu)=\nu,
\end{aligned}
$$

here the subscript + , - just indicates the position inside or outside the unit circle.

In terms of time variables, the action of loop group elements represented in the formula (140) (taking into account the factorization formulae) looks 
like

$$
\begin{aligned}
& g_{\left(\lambda_{+}, \mu_{+}\right)} \rightarrow \mathbf{x}_{+}+\left[\mu_{+}\right]-\left[\lambda_{+}\right], \\
& g_{\left(\lambda_{-}, \mu_{-}\right)} \rightarrow \mathbf{x}_{-}+\left[\mu_{-}^{-1}\right]-\left[\lambda_{-}^{-1}\right], \\
& g_{\left(\lambda_{+}, \mu_{-}\right)} \rightarrow n+1, \mathbf{x}_{+}-\left[\lambda_{+}\right], \mathbf{x}_{-}+\left[\mu_{-}^{-1}\right], \\
& g_{\left(\lambda_{-}, \mu_{+}\right)} \rightarrow n-1, \mathbf{x}_{+}+\left[\mu_{+}\right], \mathbf{x}_{-}-\left[\lambda_{-}^{-1}\right] .
\end{aligned}
$$

Substituting these formulae to (140), we obtain a standard form of addition formulae for the Toda lattice hierarchy. It occurs that off-diagonal components of (140) give the same formula with interchanged $\lambda, \mu$, so we have three addition formulae

$$
\begin{aligned}
& \lambda \tau_{n}^{\left(x_{+}+[\mu], x_{-}\right)} \tau_{n}^{\left(x_{+}, x_{-}+\left[\lambda^{-1}\right]\right)}-\mu \tau_{n-1}^{\left(x_{+}+[\mu], x_{-}\right)} \tau_{n+1}^{\left(x_{+}, x_{-}+\left[\lambda^{-1}\right]\right)}= \\
& (\lambda-\mu) \tau_{n}^{\left(x_{+}, x_{-}\right)} \tau_{n}^{\left(x_{+}+[\mu], x_{-}+\left[\lambda^{-1}\right]\right)}, \\
& |\lambda|>1,|\mu|<1, \\
& \lambda \tau_{n+1}^{\left(x_{+}+[\mu], x_{-}\right)} \tau_{n}^{\left(x_{+}+[\lambda], x_{-}\right)}-\mu \tau_{n}^{\left(x_{+}+[\mu], x_{-}\right)} \tau_{n+1}^{\left(x_{+}+[\lambda], x_{-}\right)}= \\
& (\lambda-\mu) \tau_{n+1}^{\left(x_{+}, x_{-}\right)} \tau_{n}^{\left(x_{+}+[\mu]+[\lambda], x_{-}\right)}, \\
& |\lambda|<1,|\mu|<1, \\
& \lambda \tau_{n}^{\left(x_{+}, x_{-}+\left[\mu^{-1}\right]\right)} \tau_{n-1}^{\left(x_{+}, x_{-}+\left[\lambda^{-1}\right]\right)}-\mu \tau_{n-1}^{\left(x_{+}, x_{-}+\left[\mu^{-1}\right]\right)} \tau_{n}^{\left(x_{+}, x_{-}+\left[\lambda^{-1}\right]\right)}= \\
& (\lambda-\mu) \tau_{n-1}^{\left(x_{+}, x_{-}\right)} \tau_{n}^{\left(x_{+}, x_{-}+\left[\mu^{-1}\right]+\left[\lambda^{-1}\right]\right)}, \\
& |\lambda|>1,|\mu|>1 .
\end{aligned}
$$

In fact, we have used only the first equation of the pair of equations (118), but the second equation gives exactly the same set of addition formulae.

The formula (143) is a well-known simplest addition formula for the Toda lattice hierarchy. The formulae (144), (145) do not shift negative (positive) times and it is connected with the Bäcklund transformations for the KP equation.

To get more generic addition formulae, one could use the formula (110). To get more symmetric expressions, we will use a special version of this formula corresponding to the choice

$$
g_{0}(\nu):=g_{1}(\nu) g_{2}^{-1}(\nu)=\frac{\nu-\xi}{\nu-\eta}
$$


where we do not specify from the beginning the position of the points $\lambda_{1}, \mu_{1}$. This choice leads to the following functional form of the addition formula

$$
\begin{aligned}
& (\mu-\eta)(\lambda-\xi) \tau\left(g g_{(\xi, \eta)} g_{(\lambda, \mu)}\right) \tau(g)-(\lambda-\eta)(\mu-\xi) \tau\left(g g_{(\lambda, \mu)}\right) \tau\left(g g_{(\xi, \eta)}\right)+ \\
& (\lambda-\mu)(\eta-\xi) \tau\left(g g_{(\xi, \mu)}\right) \tau\left(g g_{(\lambda, \eta)}\right)=0 .
\end{aligned}
$$

The formula (147) contains 16 components depending on the position of four parameters with respect to unit circle. It is convenient to introduce the function showing the position of each parameter

$$
i_{\lambda}=\left\{\begin{array}{cc}
\frac{1}{2} & |\lambda|<1 \\
-\frac{1}{2} & |\lambda|>1
\end{array}\right.
$$

Then the addition formulae take the form

$$
\begin{aligned}
& (\mu-\eta)(\lambda-\xi) \tau_{n-i_{\eta}-i_{\mu}}^{(\mathbf{x}+[[\eta]]+[[\mu]])} \tau_{n-i_{\lambda}-i \xi}^{(\mathbf{x}+[[\lambda]]+[[\xi]])}- \\
& (\lambda-\eta)(\mu-\xi) \tau_{n-i_{\mu}-i_{\xi}}^{(\mathbf{x}+[[\xi]]+[[\mu]])} \tau_{n-i_{\lambda}-i_{\eta}}^{(\mathbf{x}+[[\lambda]]+[[\eta]])}+ \\
& (\lambda-\mu)(\eta-\xi) \tau_{n-i_{\lambda}-i_{\mu}}^{(\mathbf{x}+[[\mu]]+[[\lambda]])} \tau_{n-i_{\eta}-i_{\xi}}^{(\mathbf{x}+[[\eta]]+[[\xi]])}=0,
\end{aligned}
$$

where

$$
(\mathbf{x}+[[\lambda]])= \begin{cases}\left(x_{+}+[\lambda], x_{-}\right) & |\lambda|<1 \\ \left(x_{+}, x_{-}+\left[\lambda^{-1}\right]\right) & |\lambda|>1\end{cases}
$$

Due to its symmetric form, the formula (149) contains only five different addition formulae corresponding to all four parameters inside the unit circle, one parameter outside, two parameters outside, three parameters outside, all four parameters outside. The first and the last case just give the KP addition formulae, so we will consider only remaining three cases. First we write down the addition formulae for the case when two variables (say, $\lambda$ and $\mu)$ are inside the unit circle:

$$
\begin{aligned}
& (\mu-\eta)(\lambda-\xi) \tau_{n}^{\left(\mathbf{x}_{+}+[\mu], \mathbf{x}_{-}+\left[\eta^{-1}\right]\right)} \tau_{n}^{\left(\mathbf{x}_{+}+[\lambda], \mathbf{x}_{-}+\left[\xi^{-1}\right]\right)}- \\
& (\lambda-\eta)(\mu-\xi) \tau_{n}^{\left(\mathbf{x}_{+}+[\mu], \mathbf{x}_{-}+\left[\xi^{-1}\right]\right)} \tau_{n}^{\left(\mathbf{x}_{+}+[\lambda], \mathbf{x}_{-}+\left[\eta^{-1}\right]\right)}+ \\
& (\lambda-\mu)(\eta-\xi) \tau_{n-1}^{\left(\mathbf{x}_{+}+[\mu]+[\lambda], \mathbf{x}_{-}\right)} \tau_{n+1}^{\left(\mathbf{x}_{+}, \mathbf{x}_{-}+\left[\eta^{-1}\right]+\left[\xi^{-1}\right]\right)}=0 .
\end{aligned}
$$

The structure of this addition formula is similar to the formula (143), and in fact (150) is a generalized version of (143). 
When one parameter (say, $\lambda$ ) is inside the unit circle, we obtain the following addition formula

$$
\begin{aligned}
& (\mu-\eta)(\lambda-\xi) \tau_{n+1}^{\left(\mathbf{x}_{+}, \mathbf{x}_{-}+\left[\eta^{-1}\right]+\left[\mu^{-1}\right]\right)} \tau_{n}^{\left(\mathbf{x}_{+}+[\lambda], \mathbf{x}_{-}+\left[\xi^{-1}\right]\right)}- \\
& (\lambda-\eta)(\mu-\xi) \tau_{n+1}^{\left(\mathbf{x}_{+}, \mathbf{x}_{-}+\left[\xi^{-1}\right]+\left[\mu^{-1}\right]\right)} \tau_{n}^{\left(\mathbf{x}_{+}+[\lambda], \mathbf{x}_{-}+\left[\eta^{-1}\right]\right)}+ \\
& (\lambda-\mu)(\eta-\xi) \tau_{n}^{\left(\mathbf{x}_{+}+[\lambda], \mathbf{x}_{-}+\left[\mu^{-1}\right]\right)} \tau_{n+1}^{\left(\mathbf{x}_{+}, \mathbf{x}_{-}+\left[\eta^{-1}\right]+\left[\xi^{-1}\right]\right)}=0 .
\end{aligned}
$$

And the last case ( $\lambda$ outside the unit circle) gives

$$
\begin{aligned}
& (\mu-\eta)(\lambda-\xi) \tau_{n-1}^{\left(\mathbf{x}_{+}+[\eta]+[\mu], \mathbf{x}_{-}\right)} \tau_{n}^{\left(\mathbf{x}_{+}+[\xi], \mathbf{x}_{-}+\left[\lambda^{-1}\right]\right)}- \\
& (\lambda-\eta)(\mu-\xi) \tau_{n-1}^{\left(\mathbf{x}_{+}+[\xi]+[\mu], \mathbf{x}_{-}\right)} \tau_{n}^{\left(\mathbf{x}_{+}+[\eta], \mathbf{x}_{-}+\left[\lambda^{-1}\right]\right)}+ \\
& (\lambda-\mu)(\eta-\xi) \tau_{n}^{\left(\mathbf{x}_{+}+[\mu], \mathbf{x}_{-}+\left[\lambda^{-1}\right]\right)} \tau_{n-1}^{\left(\mathbf{x}_{+}+[\eta]+[\xi], \mathbf{x}_{-}\right)}=0 .
\end{aligned}
$$

The formulae (151), (152) generalize the formulae (144), (145).

\section{Acknowledgments}

The first author (LB) is grateful to the Dipartimento di Fisica dell'Università and Sezione INFN, Lecce, for hospitality and support; (LB) also acknowledges partial support from the Russian Foundation for Basic Research under grant No 96-01-00841 and by INTAS under grant 93-166.

\section{References}

[1] M. Sato,RIMS, Kokyuroku, Kyoto Univ. 439, 30 (1981)

M. Sato and Y. Sato, in Nonlinear partial differential equations in applied science (Proc. U.S. - Japan Seminar, Tokyo, 1982) (Amsterdam New York: North Holland, 1983).

[2] M. Jimbo and T. Miwa, Publ. RIMS, Kyoto Univ. 19, 943 (1983)

[3] E. Date, M. Kashiwara, M. Jimbo and T. Miwa, Transformation groups for soliton equations, in Non-linear integrable equations - classical and quantum theory, M. Jimbo and T. Miwa (Eds) (World Scientific, Singapore, 1983)

[4] K. Ueno and K. Takasaki, Adv. Studies Pure Math. 1, 4 (1984) 
[5] K. Takasaki, Rev. Math. Phys. 1, 1 (1989)

[6] G. Segal and G. Wilson, Publ. Math. I.H.E.S. 61, 1 (1985)

[7] V.E Zakharov and S.V. Manakov, Zap. N. S. LOMI 133, 77 (1984), V.E. Zakharov and S.V. Manakov, Funk. Anal. Ego Prilozh. 19, 11 (1985).

[8] L.V. Bogdanov and S.V. Manakov, J. Phys. A.:Math. Gen. 21, L537 (1988).

[9] V.E. Zakharov, in: Inverse Methods in Action, ed P C Sabatier (Berlin: Springer, 1990)

[10] B.G. Konopelchenko, Solitons in multidimensions (World Scientific, Singapore, 1993)

[11] L. V. Bogdanov and B. G. Konopelchenko, J. Phys. A: Math. Gen. 30, 1591 (1997).

[12] L. V. Bogdanov and B. G. Konopelchenko, Analytic-bilinear approach to integrable hierarchies. I. Generalized KP hierarchy., preprint solvint/9609009, (1996)

[13] L.V. Bogdanov, Physica D 87, 58 (1995).

[14] P.G. Grinevich, A.Yu. Orlov, in Modern problems of quantum field theory (Springer-Verlag, 1989)

[15] V.G. Kac and J.W. Van de Leur, in: Important developments in soliton theory (A.S. Fokas and V.E. Zakharov, Eds) (Springer-Verlag, Berlin, 1993), p. 302

[16] L.A. Dickey, Soliton equations and Hamiltonian systems (World Scientific, Singapore, 1991)

[17] F. Nijhoff and H.W. Capel, Inverse Problems 6, 567 (1990)

[18] E. Ferapontov, Teor. i Mat. Fiz. 110, 86 (1997)

[19] A.B. Shabat and R.T. Yamilov, Phys. Lett. A 227, 15 (1997) 
[20] J.D. Gibbon and M. Tabor, J. Math. Phys. 26, 1956 (1985)

[21] G. Darboux, Lecons sur les systémes orthogonaux et les coordonneés curvilignes (Paris, 1910).

[22] B.G. Konopelchenko, Rev. Math. Phys. 2, 399 (1990)

[23] V.E. Zakharov, S.V. Manakov, S.P. Novikov and L.P. Pitaevsky Theory of solitons (Consultant Bureau, New York, 1984)

[24] L.V.Bogdanov, Teor. i Mat. Fiz. 70, 309 (1987)

[25] L.P. Nizhnik, DAN SSSR 254, 332 (1980)

[26] A.P. Veselov and S.P. Novikov, DAN SSSR 279, 20 (1984)

[27] T. Shiota, Invent. Math. 83, 333 (1986) 